\title{
An overview on managing additive consistency of reciprocal preference relations for consistency-driven decision making and Fusion:
}

\section{Taxonomy and future directions}

\author{
Cong-Cong $\mathrm{Li}^{\mathrm{a}, \mathrm{b}}$, Yucheng Dong ${ }^{\mathrm{a}, *}$, Yejun $\mathrm{Xu}^{\mathrm{c}}$, Francisco Chiclana ${ }^{\mathrm{d}, \mathrm{b}}$, \\ Enrique Herrera-Viedma ${ }^{\mathrm{b}, \mathrm{e}}$, Francisco Herrera ${ }^{\mathrm{b}, \mathrm{f}}$ \\ a. Business School, Sichuan University, Chengdu, China \\ b. Andalusian Research Institute on Data Science and Computational Intelligence (DaSCI), \\ University of Granada, 18071, Granada, Spain \\ c. Business School, Hohai University, Nanjing, China \\ d. Institute of Artificial Intelligence, De Montfort University, Leicester, UK \\ e. Department of Electrical and Computer Engineering, Faculty of Engineering, King Abdulaziz University, \\ Jeddah, Saudi Arabia \\ f. Faculty of Computing and Information Technology, King Abdulaziz University, Jeddah, Saudi Arabia
}

\begin{abstract}
The reciprocal preference relation (RPR) is a powerful tool to represent decision makers' preferences in decision making problems. In recent years, various types of RPRs have been reported and investigated, some of them being the 'classical' RPRs, interval-valued RPRs and hesitant RPRs. Additive consistency is one of the most commonly used property to measure the consistency of RPRs, with many methods developed to manage additive consistency of RPRs. To provide a clear perspective on additive consistency issues of RPRs, this paper reviews the consistency measurements of the different types of RPRs. Then, consistency-driven decision making and information fusion methods are also reviewed and classified into four main types: consistency improving methods; consistency-based methods to manage incomplete RPRs; consistency control in consensus decision making methods; and consistency-driven linguistic decision making methods. Finally, with respect to insights gained from prior researches, further directions for the research are proposed.
\end{abstract}

Keywords: Decision making; Reciprocal preference relation; Additive consistency; Consistency-driven method

\footnotetext{
${ }^{*}$ Corresponding author.

Email adresses: congcongli@correo.ugr.es(C. Li), ycdong@scu.edu.cn (Y. Dong), xuyejohn@163.com (Y. Xu), chiclana@dmu.ac.uk (F. chiclana), viedma@decsai.ugr.es (E. Herrera Viedma), herrera@decsai.ugr.es (F. Herrera).
} 


\section{Introduction}

Decision making is a problem-solving activity, which involves the process of identifying and choosing alternatives based on the preferences of decision makers. The concept of preference relation, which is constructed by comparing alternatives using pre-established scales, is one of the most commonly used representation formats of decision makers' preferences. Various types of reciprocal preference relation (RPRs) have been developed: additive preference relation (APR) [1-5] (also called reciprocal fuzzy preference relation), multiplicative preference relation (MPR) [6-9], linguistic preference relation (LPR) [10-13]. The elements of the APR, MPR and LPR are represented by a crisp number or single linguistic term. In some complex decision making situations, it is evident that decision makers may be unable to describe 'the exact degree of preferences' between pairs of alternatives and they would prefer the use of interval values or more than one discrete value to express their preferences. Thus, the interval-valued preference relations [14-16] and hesitant preference relations [18] are of practical interest.

Measuring consistency is an important issue in decision making with RPRs to ensure that decision makers are neither random nor illogical when providing their preferences; otherwise, lack of consistency may lead to inconsistent and unreasonable conclusions. In the research context of interest of this paper, consistency is usually associated with the notion of transitivity. However, there are many different transitivity conditions that can be used to examine the consistency of RPRs [1,5,19-21]: weak transitivity, max-min transitivity, max$\max$ transitivity, restricted $\max -\min$ transitivity, restricted max-max transitivity, multiplicative transitivity and additive transitivity. In particular, additive transitivity has proved to be a most popular tool among researchers for developing RPRs consistency measures and corresponding consistency-driven approaches to solve decision making problems with different aims and objectives. A first aim of this paper is to present a classification of the existing consistency-driven approaches into four main types: consistency improving methods [22,23]; consistency-based methods to manage incomplete RPRs [21,24,25]; consistency control in consensus decision making methods [26,27]; and consistency-driven linguistic decision making methods [28,29]. A second aim of the present paper is to provide a coherence approach on how the use of additive consistency in decision making with RPRs by providing a comprehensive review of both the additive consistency measures proposed in the literature for the different types of RPRs: APR, MPR, LPR, 
interval-valued RPR and hesitant RPR; and the different consistency-driven decision methods with RPRs. As a by-product of the analysis of prior research, further research directions are also discussed.

The remainder of the paper is organized as follows: Section 2 proposes a taxonomy of RPRs and introduces the different additive consistency measures proposed in the literature. In Section 3, existing consistency-driven methods in decision making are presented. Section 4 analyses the known consistency studies of RPRs and proposes research directions to develop this are further. Finally, Section 5 draws the conclusion of this paper.

\section{Additive consistency measures of reciprocal preference relations}

In this section, we present a taxonomy of the various types of RPRs followed by a review of the additive consistency measures proposed for each type of RPR.

\subsection{A taxonomy for reciprocal preference relations and additive consistency measurement}

Let $X=\left\{x_{1}, x_{2}, \ldots, x_{n}\right\}$ be a finite set of alternatives. When a decision maker makes pairwise comparisons, he/she construct an RPR, which depending on the domain used to modelled the preference elements can be of the following types:

(1) Classical RPRs with crisp/precise numerical elements. This type of RPRs includes both the APR [1] (also called reciprocal fuzzy preference relation), the MPR [30] and the LPR $[12,13]$.

(2) Interval-valued RPRs, which include the interval-valued additive preference relation (IVAPR) [14] (also called interval-valued fuzzy preference relation), the interval-valued multiplicative preference relation (IVMPR) [31] and the interval-valued linguistic preference relation (IVLPR) [32].

(3) Hesitant RPRs, which includes the hesitant APR [18], the hesitant MPR and the hesitant fuzzy linguistic preference relation (HFLPR) [17].

Next, an analysis of the basic ideas used in the existing studies for measuring additive consistency for the three types of RPRs is provided.

The basic ideas used in measuring consistency for classical RPRs (i.e., APR, MPR and LPR) are similar. Consistent preference values are estimated from the elements of an RPR using the additive transitivity property [20]. By computing the difference (error) between the RPR preference values and the estimated consistent preference values, the RPR consistency degree can be measured. Two different ways to measure the consistency degree of a classical 
RPR have been proposed.

- Local method. In this method, the consistent pairwise preference values are locally estimated. Then, the consistency degree of an RPR can be measured by analyzing the difference between the pairwise preference values and the estimated values.

- Global method. In this method, a priority vector or a consistent RPR is estimated via optimization models. Then, the consistency degree of an RPR can be measured by analyzing the difference between the RPR and the derived priority vector or the consistent RPR, respectively.

The methods used in the consistency measurements for interval-valued RPRs and hesitant RPRs are based on similar ideas to the ones used for RPRs. This is because both the interval-valued RPR and the hesitant RPR can be interpreted as a collection of classical RPRs, and thus their consistency degree can be analyzed by measuring the associated classical RPRs $[15,33]$. For example, an interval-valued additive RPR can be seen as the collection of additive RPRs, and its consistency can be determined by the consistency status of these associated additive RPRs.

Fig.1 shows the taxonomy for RPRs and additive consistency measurement.

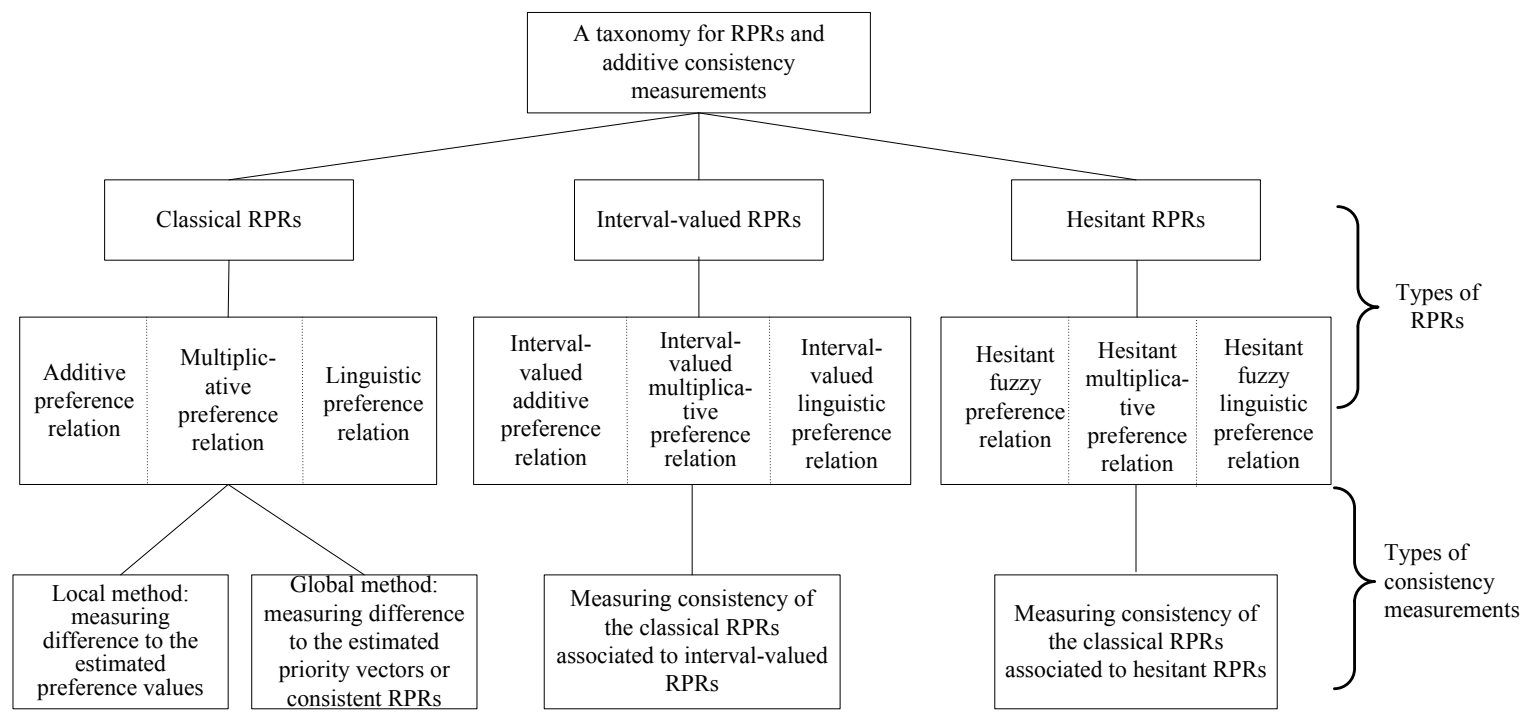

Fig.1 A taxonomy for RPRs and additive consistency measurements

\subsection{Consistency measurement in classical RPRs}

We introduce the consistency measurement of APRs, MPRs and LPRs in this subsection.

\subsubsection{Additive preference relation}

In decision making problems, when decision makers make pairwise comparison between alternatives using crisp numerical values in the domain [0,1], an APR is constructed. The 
concept of APR is provided as Definition 1.

Definition 1 [20]. An APR on a set of alternatives $X$ is represented by a matrix $F=\left(f_{i j}\right)_{n \times n}$, with $f_{i j}$ in $[0,1]$ being interpreted as the preference intensity of alternative $x_{i}$ to that of $x_{j}$, satisfying the following reciprocity property: $f_{i j}+f_{j i}=1$ for $i, j=1,2, \ldots, n$.

Considering the value $f_{i j}-0.5$ as an intensity of preference of $x_{i}$ over $x_{j}$, the additive transitivity property of intensities of preferences is defined as follows [7]: $\left(f_{i j}-0.5\right)+\left(f_{j k}-0.5\right)=\left(f_{i k}-0.5\right) \quad$ for $i, j, k=1,2, \ldots, n$

If the preference values in $F=\left(f_{i j}\right)_{n \times n}$ fulfill Eq. (1), then $F$ is considered additive consistent. This is summarized in the following Definition 2.

Definition 2 [20]. An APR $F=\left(f_{i j}\right)_{n \times n}$ is additive consistent if $f_{i j}=f_{i k}+f_{k j}-0.5 \quad$ for $i, j, k=1,2, \ldots, n$

Based on Eq. (2), a consistent preference value associated to the pair of alternatives $\left(x_{i}, x_{j}\right)$ can be estimated by the value of $f_{j k}+f_{i k}-0.5$. Thus, by analyzing the difference between preference values $f_{i j} \quad(i, j=1,2, \ldots, n)$ and its estimated value $f_{j k}+f_{i k}-0.5$ $(i, j, k=1,2, \ldots, n)$, in [21] the following consistency measurement for an APR $F$ is presented.

Definition 3 [21]. The consistency index of an APR $F$ is

$$
C I(F)=1-\frac{2}{3 n(n-1)(n-2)} \sum_{i, j, k=1 ; i \neq j \neq k}^{n}\left|f_{i j}+f_{j k}-f_{i k}-0.5\right|
$$

Clearly, $C I(F) \in[0,1]$. When $C I(F)=1$, the APR $F$ is fully consistent; otherwise, the lower $C I(F)$ the more inconsistent is $F$.

A priority vector $w=\left(w_{1}, w_{2}, \ldots, w_{n}\right)$ reflects the importance degree of the alternatives $X=\left(x_{1}, x_{2}, \ldots, x_{n}\right)$ and, consequently, the following consistency measurement based on the priority vector for the APR $F$ has also been proposed in the literature.

Definition $4[20,34]$. Let $w=\left(w_{1}, w_{2}, \ldots, w_{n}\right)$ be a priority vector that satisfies $\sum_{i=1}^{n} w_{i}=1$ and $w_{i} \geq 0$ for $i=1,2, \ldots, n$. The APR $F$ is additive consistent if it satisfies $f_{i j}=0.5\left(w_{i}-w_{j}+1\right) \quad$ for $i, j=1,2, \ldots, n$

Eq. (4) can be used to optimize the priority vector of an APR by solving Model (5) : 
$\left\{\begin{array}{l}\min J=\sum_{i, j=1}^{n}\left|f_{i j}-0.5\left(w_{i}-w_{j}+1\right)\right| \\ \text { s.t. } \quad w_{i} \geq 0, \quad i=1,2, \ldots, n \\ \sum_{i=1}^{n} w_{i}=1\end{array}\right.$

When $J=0, F$ is an additive consistent preference relation; otherwise the lower the value of $J$ the more consistent is $F$. The consistency measurement in [21] and [20] can be considered to be local and the global methods, respectively.

\subsubsection{Multiplicative preference relation}

The MPR is widely used in the analytic hierarchy process (AHP), and is defined as follows:

Definition 5 [7]. A MPR on a set of alternatives $X$ is represented by a matrix $A=\left(a_{i j}\right)_{n \times n}$, with element $a_{i j}$ being interpreted as the ratio of the preference intensity of alternative $x_{i}$ to that of $x_{j}$, and verifying the following reciprocity property $a_{i j} \cdot a_{j i}=1$, for $i, j=1,2, \ldots, n$.

Given an MPR the cardinal transitivity property of intensities of preferences is presented as follows [7],

$a_{i j} \times a_{j k}=a_{i k}$

for all $i, j, k=1, \ldots, n$.

It has been proved that an MPR $A$ is cardinal transitive if there exists a priority vector $w=\left(w_{1}, w_{2}, \ldots, w_{n}\right)$ satisfying

$a_{i j}=w_{i} / w_{j}$ for $i, j=1,2, \ldots, n$.

Notably, there exist some transformation functions between MPR and APR [34,44], and the transitivity property in Eq. (6) or Eq. (7) is equivalent to additive transitivity of APR when transforming an MPR into an APR based on the transformation functions.

The popular measurements of consistency of the MPR are based on measuring the difference between the MPR and the derived priority vector, and therefore can be categorized as global methods. Saaty $[7,30]$ proposed the principle eigenvector method to determine the desired priority vector of an MPR, which can be obtained by solving the linear system, $A w=\lambda_{\max } w$, where $\lambda_{\max }$ is the largest or principal eigenvalue of $A$. A consistency measurement with the principal eigenvalue was defined in [7] as $C I(A)=\frac{\lambda_{\max }-n}{n-1}$, which is 
zero when, i.e. when $A$ is fully consistent.

To overcome the dependency of $n$, Satty [7] proposed a normalized measure via the consistency ratio $C R=\frac{C I}{R I}$, where $R I$ is the mean consistency index of randomly generated MPRs. If $C R<0.1$, then MPR is of acceptable consistency.

Crawford and Williams [6] provided the row geometric mean method by solving an optimization-based model to obtain the priority vector of MPR $A$,

$$
\left\{\begin{array}{l}
\min \sum_{i=1}^{n} \sum_{i<j}\left(\log \left(a_{i j}\right)-\left(\log \left(w_{i}\right)-\log \left(w_{j}\right)\right)\right)^{2} \\
\text { s.t. } \sum_{i=1}^{n} w_{i}=1, w_{i} \geq 0
\end{array}\right.
$$

They showed that the optimal solution of the above model is unique and can be expressed as [6],

$$
w_{i}=\frac{\sqrt[n]{\prod_{j=1}^{n} a_{i j}}}{\sum_{l=1}^{n} \sqrt[n]{\prod_{j=1}^{n} a_{l j}}}, i=1,2, \ldots, n
$$

The geometric consistency measurement of MPR is therefore presented below in Definition 6.

Definition 6 [6]. Let $A=\left(a_{i j}\right)_{n \times n}$ be an MPR, and $w=\left(w_{1}, w_{2}, \ldots, w_{n}\right)$ be the priority vector derived by the row geometric mean method. The geometric consistency index (GCI) of $A$ is defined by

$$
G C I(A)=1-\frac{2}{(n-1)(n-2)} \sum_{i, j=1, i<j}^{n}\left(\log \left(a_{i j}\right)-\log \left(w_{i}\right)+\log \left(w_{j}\right)\right)^{2}
$$

The MPR $A$ is fully consistent if $G C I(A)=0$. Aguarón and Moreno-Jiménez [35] provided the following threshold values $\overline{G C I}$ of $G C I$ : $\overline{G C I}=0.31$ for $n=3$; $\overline{G C I}=0.35$ for $n=4$ and $\overline{G C I}=0.37$ for $n>4$. When $G C I(A)<\overline{G C I}$, the MPR is considered of acceptable consistency.

\subsubsection{Linguistic preference relation}

In certain real decision-making situations, decision makers often feel more comfortable by expressing their knowledge and preferences linguistically. The basic notations and operational laws of linguistic variables were introduced in [36]. Let $S=\left\{s_{j} \mid j=0, \cdots, g\right\}$ be a linguistic term set with odd granularity $g+1$, where the term $s_{j}$ represents a possible value 
for a linguistic variable. The linguistic term set is usually required to satisfy the following additional characteristics:

(1) The set is ordered: $s_{i} \leq s_{j}$ if and only if $i \leq j$;

(2) There is a negation operator: $\operatorname{Neg}\left(s_{j}\right)=s_{g-j}$.

Solving a decision problem with linguistic information implies the need for computing with words [37-40]. In particular, Herrera and Martínez [41] proposed the 2-tuple linguistic representation model, which represents the linguistic information by a 2-tuple $\left(s_{i}, \alpha\right) \in \bar{S}=S \times[-0.5,0.5)$, where $s_{i} \in S$ and $\alpha \in[-0.5,0.5)$. Let $\beta \in[0, g]$ be a value representing the result of a symbolic aggregation operation. The 2-tuple that expresses the equivalent information to $\beta$ is then obtained as:

$$
\Delta:[0, g] \rightarrow \bar{S}
$$

being

$$
\Delta(\beta)=\left(s_{i}, \alpha\right), \text { with }\left\{\begin{array}{l}
s_{i}, i=\operatorname{round}(\beta) \\
\alpha=\beta-i, \alpha \in[-0.5,0.5)
\end{array}\right.
$$

Function $\Delta$ is a one to one mapping whose inverse function $\Delta^{-1}: \bar{S} \rightarrow[0, g]$ is defined as $\Delta^{-1}\left(s_{i}, \alpha\right)=i+\alpha$. A 2 -tuple $\left(s_{i}, \alpha\right)$ with $\alpha=0$ is called a simple term.

LPRs are widely used in decision making, and are defined as Definition 7.

Definition $7[12,13]$. Let $S=\left\{s_{0}, s_{1}, \ldots, s_{g}\right\}$ be a linguistic term set. A LPR on a set of alternatives $X=\left\{x_{1}, x_{2}, \ldots, x_{n}\right\}$ is represented by a matrix $L=\left(l_{i j}\right)_{n \times n}$, whose element $l_{i j} \in S$ estimates the preference degree of alternative $x_{i}$ over $x_{j}$, and satisfies $l_{i j}=\operatorname{Neg}\left(l_{j i}\right)$ for $i, j=1,2, \ldots, n$.

In computing with the linguistic preference values of LPRs, some transformation functions between linguistic terms and numerical values are presented. In this review the 2tuple linguistic model function $\Delta^{-1}$ is applied to transform the linguistic terms into numerical values: $\Delta^{-1}\left(s_{i}\right)=i$ and $\Delta^{-1}\left(s_{i}, \alpha\right)=i+\alpha$ with $\alpha \in[-0.5,0.5]$.

In the LPR $L=\left(l_{i j}\right)_{n \times n}$, the following additive transitivity property of intensities of linguistic preferences is presented [42],

$$
\left(\Delta^{-1}\left(l_{i j}\right)-\frac{g}{2}\right)+\left(\Delta^{-1}\left(l_{j k}\right)-\frac{g}{2}\right)=\left(\Delta^{-1}\left(l_{i k}\right)-\frac{g}{2}\right) \quad \text { for } i, j, k=1,2, \ldots, n,
$$

The concept of consistent LPR based on additive transitivity is presented as in the below 
Definition 8.

Definition 8 [42]. Let $L=\left(l_{i j}\right)_{n \times n}$ be a linguistic preference relation based on a linguistic term set $S . L$ is consistent if

$\Delta^{-1}\left(l_{i j}\right)+\Delta^{-1}\left(l_{j k}\right)-\Delta^{-1}\left(l_{i k}\right)=\frac{g}{2}$ for $i, j, k=1,2, \ldots, n$.

Based on Eq. (13), by measuring the difference between the preference value $\Delta^{-1}\left(l_{i j}\right)$ and its estimated value $\Delta^{-1}\left(l_{i k}\right)+\Delta^{-1}\left(l_{k j}\right)-\frac{g}{2}(i, j, k=1,2, \ldots, n)$, a consistency measurement for an LPR is provided as follows.

Definition 9 [42]. Let $L=\left(l_{i j}\right)_{n \times n}$ be an LPR, the consistency index of $L$, using Manhanttan distance, is

$$
C I(L)=1-\frac{2}{3 g n(n-1)(n-2)} \sum_{i, j, k=1 ; i \neq j \neq k}^{n}\left|\Delta^{-1}\left(l_{i j}\right)+\Delta^{-1}\left(l_{j k}\right)-\Delta^{-1}\left(l_{i k}\right)-\frac{g}{2}\right|
$$

The larger the value of $C I(L)$ the more consistent $L$ is. When $C I(L)=1, L$ is a consistent LPR.

The above is not the unique measure proposed in literature for measuring consistency of an LPR. The following definition provides an alternative measure based on the difference between an LPR and its consistent LPR.

Definition $10[10,11]$. Let $L=\left(l_{i j}\right)_{n \times n}$ be an LPR and $M_{n}$ be the set of $n \times n$ consistent LPRs, and

$$
d\left(L, \bar{L}^{*}\right)=\min _{L \in M_{n}} d(L, \bar{L})
$$

the minimal distance between L and $M_{n}$. The consistency index of the LPR L is

$$
C I(L)=1-d\left(L, \bar{L}^{*}\right)=1-\frac{1}{n(n-1)} \sum_{i<j}\left(\frac{\left|\Delta^{-1}\left(l_{i j}\right)-\Delta^{-1}\left(\bar{l}_{i j}^{*}\right)\right|}{g}\right)
$$

In [10], based on Eq. (13), the following optimized consistent LPR $\bar{L}^{*}=\left(\bar{l}_{i j}^{*}\right)_{n \times n} \in M_{n}$ is constructed from $L$

$$
\Delta^{-1}\left(\bar{l}_{i j}^{*}\right)=\frac{2}{3(n-2)} \sum_{k=1 ; k \neq i \neq j}^{n}\left(\Delta^{-1}\left(l_{i k}\right)+\Delta^{-1}\left(l_{k j}\right)-g / 2\right)
$$

In [11], the following optimization-based model is proposed to obtain $\bar{L}^{*}$ with the aim of minimizing the distance between LPR and its consistent LPR, 


$$
\left\{\begin{array}{l}
\min d(L, \bar{L}) \\
\text { s.t. } \\
\Delta^{-1}\left(\overline{l_{i j}}\right)+\Delta^{-1}\left(\overline{l_{j k}}\right)-\Delta^{-1}\left(\overline{l_{i k}}\right)=\frac{g}{2}, \quad i, j, k=1,2, \ldots, n \\
\Delta^{-1}\left(\overline{l_{i j}}\right)+\Delta^{-1}\left(\overline{l_{j i}}\right)=g, \quad i, j=1,2, \ldots, n
\end{array}\right.
$$

Let $W=\left\{w_{1}, w_{2}, \ldots, w_{n}\right\}$ be a priority vector from, with $w_{i} \geq 0$ and $\sum_{i=1}^{n} w_{i}=1$. As a generation of Eq. (4), the LPR is additive consistent iif it satisfies [43],

$\frac{\Delta^{-1}\left(l_{i j}\right)}{g}=0.5\left(w_{i}-w_{j}+1\right)$.

Based on Eq. (19), an optimization model can be constructed to verify the consistency of an LPR [43],

$$
\left\{\begin{array}{l}
\min \sum_{i, j=1 ; i \neq j}^{n}\left|\frac{\Delta^{-1}\left(l_{i j}\right)}{g}-0.5\left(w_{i}-w_{j}+1\right)\right| \\
\text { s.t. } \quad w_{i} \geq 0, \quad i=1,2, \ldots, n \\
\sum_{i=1}^{n} w_{i}=1
\end{array}\right.
$$

The consistency measurements that derive from Eqs. (18) and (20) can be considered to be local and the global methods, respectively. In [10], Dong et al. discussed how to establish consistency threshold values for LPRs.

\subsection{Consistency measurements in interval-valued RPRs}

Due to the complexity and uncertainty of some decision making problems, decision makers may prefer to express their preference using interval values rather than with precise judgments. As stated in Section 2.1, the interval-valued RPR includes the IVAPR, IVMPR and IVLPR. Considering that the consistency measurement of these three types of intervalvalued RPRs are based on measuring the consistency of their associated RPRs, and the transformation function between these types of RPRs have been reviewed in Chen et al. [44], in this section we focus mainly on reviewing the additive consistency measurements of IVAPR, and a brief summary is provided of the consistency measurements for IVMPR and IVLPR.

The concept of IVAPR is defined as below:

Definition 11 [14]. An IVAPR on a set of alternatives $X=\left\{x_{1}, x_{2}, \ldots, x_{n}\right\}$ is represented by a 
$\operatorname{matrix} \widetilde{V}=\left(\widetilde{v_{i j}}\right)_{n \times n}=\left(\left[v_{i j}^{-}, v_{i j}^{+}\right]\right)_{n \times n}$, where $v_{i j}^{-} \leq v_{i j}^{+}, v_{i i}^{-}=v_{i i}^{+}=0.5, v_{i j}^{-}+v_{j i}^{+}=1$ and $v_{i j}^{+}+v_{j i}^{-}=1$ for $i, j=1,2, \ldots, n$.

If $v_{i j}^{-}=v_{i j}^{+}$, then an IVAPR becomes an APR. Dong et al. [15] argued that an IVAPR can be seen as a collection of APRs, which was captured in the following definition.

Definition $12[15]$. Let $\widetilde{V}=\left(\widetilde{v_{i j}}\right)_{n \times n}=\left(\left[v_{i j}^{-}, v_{i j}^{+}\right]\right)_{n \times n}$ be an IVAPR. An APR $F=\left(f_{i j}\right)_{n \times n}$ satisfying

$v_{i j}^{-} \leq f_{i j} \leq v_{i j}^{+} \quad \forall i, j \in\{1,2, \ldots, n\}$

is called an APR associated to $\widetilde{V}$. The set of all APRs associated to $\widetilde{V}$ is denoted by $N_{\widetilde{V}}$.

Consistency measurements of IVAPRs in the existing studies are classified with respect to the following four aspects [15]: Optimistic consistency, pessimistic consistency, boundary consistency and average consistency.

(1) Optimistic consistency of IVAPRs

The optimistic consistency index of an IVAPR is determined by its associated APR with the best consistency degree, which is captured in the following Definition.

Definition 13 [140]. An IVAPR $\widetilde{V}=\left(\widetilde{v_{i j}}\right)_{n \times n}=\left(\left[v_{i j}^{-}, v_{i j}^{+}\right]\right)_{n \times n}$ is additive consistent if one of its associated APRs, $F=\left(f_{i j}\right)_{n \times n}$ where

$$
v_{i j}^{-} \leq f_{i j} \leq v_{i j}^{+}
$$

for $i, j=1,2, \ldots, n$ is additive consistent.

Based on Eq. (2), an IVAPR $\widetilde{V}$ is additive consistent, if $v_{i j}^{-} \leq f_{i j} \leq v_{i j}^{+}$and $f_{i j}=f_{i k}+f_{k j}-0.5$ for $i, j, k=1,2, \ldots, n$.

According to Eq. (4), let $w=\left\{w_{1}, w_{2}, \ldots, w_{n}\right\}$, where $w_{i} \geq 0$ for $i=1,2, \ldots, n$ and $\sum_{i=1}^{n} w_{i}=1$, be a priority vector. Then $\widetilde{V}$ is additive consistent if it satisfies the following condition $[14,16]$ :

$$
v_{i j}^{-} \leq 0.5\left(w_{i}-w_{j}+1\right) \leq v_{i j}^{+} \text {for } i, j=1,2, \ldots, n \text {. }
$$

Equation (23) was used to design an optimization model by introducing deviation variables to verify additive consistency of an IVAPR [14,16]. However, Xu et al. [141] 
argued that Eq. (23) is questionable, and the corrected one should be $v_{i j}^{-} \leq 0.5+\beta\left(w_{i}-w_{j}\right) \leq v_{i j}^{+}$, for $i, j=1,2, \ldots, n$.

(2) Pessimistic consistency of IVAPRs

The pessimistic consistency index of an IVAPR is determined by its associated APR with the worst consistency degree, which is captured in the following Definition.

Definition 14 [15]. Let $\widetilde{V}$ be an IVAPR and $F \in N_{\widetilde{V}}$ an APR associated to $\widetilde{V}$. The pessimistic consistency index (PCI) of the IVAPR is, $\operatorname{PCI}(\widetilde{V})=\min _{F \in N_{\tilde{V}}} C I(F)$,

where $C I(F)$ is computed based on the additive transitivity property as per Eq. (3).

(3) Boundary consistency of IVAPRs

Liu et al. [45] proposed measuring the consistency of IVAPRs based on two APRs constructed using the endpoints of IVAPR values. This approach is referred to as the boundary consistency measurement.

Definition 15 [45]. Let $\widetilde{V}=\left(\left[v_{i j}^{-}, v_{i j}^{+}\right]\right)_{n \times n}$ be an IVAPR. If the two APRs $P=\left(p_{i j}\right)_{n \times n}$ and $Q=\left(q_{i j}\right)_{n \times n}$ with

$$
p_{i j}=\left\{\begin{array}{l}
v_{i j}^{+}, \quad i<j \\
0.5, i=j \text { and } \quad q_{i j}=\left\{\begin{array}{ll}
v_{i j}^{-}, & i<j \\
0.5, & i=j \\
v_{i j}^{-}, & i>j
\end{array} v_{i j}^{+}, \quad i>j\right.
\end{array}\right.
$$

are additive consistent, then $\widetilde{V}$ is an additive consistent IVAPR.

It is worth noting that Wang [46] and Krejči [47] showed that the above definition of boundary consistency measurement does not hold under permutation of objects, which subsequently led Liu et al. [48] to extend and revise Definition 15 by proposing an additive consistent IVAPR with permutations $\sigma$ with the additional condition of the existence of a permutation $\sigma$ such that the two boundary RPRs $P$ and $Q$ are additive consistent (Eq. (2)).

Based on the description of an additive consistent APR as per Eq. (2), Wang et al. [49] extended the concept of additive consistency of APRs to the case of IVAPRs.

Definition 16 [49]. An IVAPR $\widetilde{V}=\left(\widetilde{v_{i j}}\right)_{n \times n}$ is called additive consistent, if the following additive transitivity property is satisfied

$$
\widetilde{v_{i j}} \oplus \widetilde{v_{j k}} \oplus \widetilde{v_{k i}}=\widetilde{v_{k j}} \oplus \widetilde{v_{j i}} \oplus \widetilde{v_{i k}} \text { for all } i, j, k=1,2, \ldots, n
$$

Using the operational laws of interval numbers [56], Eq. (26) can be rewritten as 


$$
\begin{aligned}
& v_{i j}^{-}+v_{j k}^{-}+v_{k i}^{-}=v_{j i}^{-}+v_{i k}^{-}+v_{k j}^{-} \\
& v_{i j}^{+}+v_{j k}^{+}+v_{k i}^{+}=v_{j i}^{+}+v_{i k}^{+}+v_{k j}^{+}
\end{aligned}
$$

Similar to Definition 16, Xu et al. [50] and Yue et al. [51] also extended the concept of an additive consistent APR described in Eq. (2) to the case of an IVAPR.

Notice that in Definition 16, the consistency of $\widetilde{V}$ is determined by the consistency of matrices $F^{1}=\left(v_{i j}^{-}\right)_{n \times n}$ and $F^{2}=\left(v_{i j}^{+}\right)_{n \times n}$; however, there is the issue of matrices $F^{1}$ and $F^{2}$ not preserving the reciprocity property and not being robust with respect to permutations of objects [46,47], which can be seen as evidence to support the claim of the use of $F^{1}$ and $F^{2}$ in measuring the consistency of the IVAPR as being unreasonable.

(4) Average consistency of IVAPRs

Instead of considering just one or two APRs associated to an IVAPR, Dong et al. [15] proposed a comprehensive measurement of the consistency of an IVAPR by considering the average consistency degree of all their associated APRs.

Definition 17 [15]. Let $\widetilde{V}=\left(\left[v_{i j}^{-}, v_{i j}^{+}\right]\right)_{n \times n}$ be an IVAPR. The average consistency index (ACI) of $\widetilde{V}$ is,

$$
A C I(\widetilde{V})=E(C I(F))
$$

where $F=\left(f_{i j}\right)_{n \times n} \in N_{\widetilde{V}}$ is the random APR associated to $\widetilde{V}$, i.e., $v_{i j}^{-} \leq f_{i j} \leq v_{i j}^{+}$, and $f_{i j}=1-f_{j i} . C I(F)$ is the consistency index of $F$ obtained via Eq. (3), and $E(C I(F))$ is the expected value of $C I(F)$. Consequently, $A C I(V)$ can be expressed as follows

$$
A C I(\widetilde{V})=1-\frac{4}{n(\mathrm{n}-1)(\mathrm{n}-2)} \sum_{i=1}^{n} \sum_{j=i+1}^{n} \sum_{k=j+1}^{n} E\left(\left|f_{i j}+f_{j k}-f_{i k}-0.5\right|\right)
$$

Furthermore, the average consistency of $\widetilde{V}$ is computed based on the assumption that $f_{i j} \in\left[v_{i j}^{-}, v_{i j}^{+}\right]$follows the normal distribution $f_{i j} \sim N\left(\frac{v_{i j}^{-}+v_{i j}^{+}}{2},\left(\frac{v_{i j}^{+}-v_{i j}^{-}}{6}\right)^{2}\right)$, which is based on the following arguments:

(1) Based on Jong [52] and Dong et al. [10], decision makers often have certain consistency tendency when making pairwise comparisons, and therefore it is assumed that 
$f_{i j}(i<j)$ relatively centralizes its domain close to $\frac{v_{i j}^{-}+v_{i j}^{+}}{2}$ and follows a normal distribution.

(2) The probability of $f_{i j}$ being distributed in the interval $\left[v_{i j}^{-}, v_{i j}^{+}\right]$should be close to 1 . According to the $3 \sigma$ principle of normally distributed variables [53], it is know that $P\left(\mu_{i j}-3 \sigma_{i j} \leq f_{i j} \leq \mu_{i j}+3 \sigma_{i j}\right) \approx 1$. Because $\mu_{i j}=\frac{v_{i j}^{-}+v_{i j}^{+}}{2}, \mu_{i j}-3 \sigma_{i j}=v_{i j}^{-}$and $\mu_{i j}+3 \sigma_{i j}=v_{i j}^{+}$, it is $\sigma_{i j}=\frac{v_{i j}^{+}-v_{i j}^{-}}{6}$.

Dong et al. [15] provided the below analytical procedure to compute the ACI value of an IVAPR:

Let $\quad \widetilde{V}=\left(\left[v_{i j}^{-}, v_{i j}^{+}\right]\right)_{n \times n}$ be an IVAPR, and let $\quad f_{i j} \sim N\left(\frac{v_{i j}^{-}+v_{i j}^{+}}{2},\left(\frac{v_{i j}^{+}-v_{i j}^{-}}{6}\right)^{2}\right)$; $\mu_{i j k}=\frac{v_{i j}^{-}+v_{i j}^{+}+v_{j k}^{-}+v_{j k}^{+}-v_{i k}^{-}-v_{i k}^{+}-1}{2} ; \quad \sigma_{i j k}=\frac{\sqrt{\left(v_{i j}^{+}-v_{i j}^{-}\right)^{2}+\left(v_{j k}^{+}-v_{j k}^{-}\right)^{2}+\left(v_{i k}^{+}-v_{i k}^{-}\right)^{2}}}{6} ;$ and $\Phi$ be the cumulative distribution function of the standard normal distribution $N(0,1)$. Then, the ACI value of $\widetilde{V}$ is expressed as follows:

$$
A C I(\widetilde{V})=1-\frac{4}{n(n-1)(n-2)} \sum_{i=1}^{n} \sum_{j=i+1}^{n} \sum_{k=j+1}^{n} A C I_{i j k}
$$

where

$$
A C I_{i j k}=\left\{\begin{array}{l}
\frac{2 \sigma_{i j k}}{\sqrt{2 \pi}}\left(e^{-\frac{\mu_{i j k}^{2}}{2 \sigma_{i j k}}}-e^{-\frac{9}{2}}\right)+\mu_{i j k}\left(1-2 \Phi\left(\frac{-\mu_{i j k}}{\sigma_{i j k}}\right)\right), \quad \text { if }-3 \leq \frac{-\mu_{i j k}}{\sigma_{i j k}} \leq 3 \\
\mu_{i j k}(\Phi(3)-\Phi(-3)), \quad \text { if } \frac{-\mu_{i j k}}{\sigma_{i j k}} \leq-3 \\
\mu_{i j k}(\Phi(-3)-\Phi(3)), \quad \text { if } \frac{-\mu_{i j k}}{\sigma_{i j k}} \geq 3
\end{array}\right.
$$

Dong et al. in [15] analyzed the internal mechanisms of the different consistency measurements of an IVAPR, and proposed that the combined use of the optimistic consistency measurement, the pessimistic consistency measurement and the average 
consistency measurement can comprehensively reflect the consistency status of IVAPRs.

Moreover, Dong et al. in [54] first proposed the optimistic and pessimistic consistency of the IVMPR by measuring the consistency of its associated MPRs. Wang et al. [31,55] and Islam et al. [56] proposed the optimistic consistency of the IVMPR based on priority vectors, which was based on the definition of an IVMPR being consistent iff one of its associated MPR is consistent, which was also the method used by Yao and $\mathrm{Hu}$ in [60] to define the concept of additive consistent IVLPR and the corresponding linguistic optimistic consistency approach. Liu in [57] provided the concept of boundary consistency of an IVMPR by measuring the consistency of the two MPRs constructed using the endpoints of the considered IVMPR, which was also the approach proposed by Zhang and Guo in [32] to define the boundary consistency of IVLPR based on its two associated 2-tuple LPRs. In [58], Zhang constructed an optimization model to derive a consistent IVMPR based on its endpoints, while Liu et al. in [59] proposed the acceptable approximation-consistency of an IVMPR based on its two associated matrices by considering the permutations of alternatives.

\subsection{Consistency measurements in hesitant RPRs}

The hesitant RPR, which includes hesitant APR, hesitant MPR and HFLPR, is often applied to manage situations when decision makers hesitate among several values in assessing alternatives. Rodriguez et al. [17] proposed the hesitant fuzzy linguistic term set (HFLTS) to enable the decision makers to express their preferences using several linguistic terms, which is widely used in linguistic decision making problems. Because the hesitant behavior is more likely to occur in linguistic contexts, in this section we mainly review the additive consistency measurements for HFLPRs. The consistency measurements of hesitant APRs and hesitant MPRs are similar to that of HFLPRs, and thus only a brief summary is provided in these cases.

The concepts of HFLTS and HFLPR are provided below:

Definition 18 [17]. Let $S=\left\{s_{0}, \ldots, S_{g}\right\}$ be a linguistic term set. A HFLTS, $H_{S}$, is an ordered finite subset of consecutive linguistic terms of $S$.

Definition 19 [17]. Let $H_{S}$ be a set of HFLTSs based on $S$. A HFLPR based on $S$ is represented by a matrix $H=\left(H_{i j}\right)_{n \times n}$, where $H_{i j} \in H_{S}$ and $\operatorname{Neg}\left(H_{i j}\right)=H_{j i}$.

A HFLPRs $H$ can be seen as a collection of associated LPRs, as defined below:

Definition 20 [33]. Let $H$ be a HFLPR. $L=\left(l_{i j}\right)_{n \times n}$ is called an LPR associated to $H$, if $l_{i j} \in H_{i j}$ and $l_{i j}=\operatorname{Neg}\left(l_{j i}\right)$. 
As mentioned in Section 2.1, consistency measurements of a HFLPR are based mainly on computing the consistency of its associated LPRs. In the following, we review the consistency measurement of HFLPRs from the same previously mentioned four aspects: Optimistic and pessimistic consistency, boundary consistency, average consistency.

(1) Optimistic and pessimistic consistency of HFLPRs

The optimistic and pessimistic consistency measurements refer to the best and worst consistency indexes of the LPRs associated to a HFLPR, respectively. If the optimistic consistency degree is equal to 1 , then there exists an additive consistent LPR associated to the HFLPR.

Li et al. [33] defined the optimistic and pessimistic consistency of HFLPRs by measuring the consistency of LPRs associated to $H$ based on Eq. (14).

Definition 21 [33]. Let $H$ be a HFLPR and $L \in N_{H}$ be the LPRs associated to $H$. The optimistic consistency index (OCI) of $H$ is,

$O C I(H)=\max _{L \in N_{H}} C I(L)$

The pessimistic consistency index (PCI) of $H$ is,

$P C I(H)=\min _{L \in N_{H}} C I(L)$

With the OCI and PCI, the interval consistency index (ICI) of $H$ can be constructed as follow,

$I C I(H)=[W C I(H), B C I(H)]$

Feng et al. [61] proposed the below linguistic geometric consistency index of a HFLPR, which in essence it is an optimistic consistency measurement of the HFLPR by measuring the LPR associated to HFLPR based on its priority vector.

Let $w=\left\{w_{1}, w_{2}, \ldots, w_{n}\right\}$ be a priority vector with $\sum_{i=1}^{n} w_{i}=1$ and $w_{i}>0$. Let $L=\left(l_{i j}\right)_{n \times n} \in N_{H}$ be the LPRs associated to $H . H$ is an additive consistent HFLPR if there exists an LPR $L$ that satisfies

$$
\frac{\Delta^{-1}\left(l_{i j}\right)}{g}=\alpha\left(w_{i}-w_{j}\right)+0.5 \quad \text { for } i, j=1,2, \ldots, n
$$

where $\alpha \geq \frac{n-1}{2}$. Based on Eq. (35), the linguistic geometric consistency index of the HFLPR $H$ is defined in [61]: 


$$
L G C I(H)=1-\frac{2}{(n-1)(n-2)} \sum_{i, j=1 ; i<j}^{n}\left(\frac{\Delta^{-1}\left(l_{i j}\right)}{g}-\alpha\left(w_{i}-w_{j}\right)-0.5\right)^{2}
$$

(2) Boundary consistency of HFLPRs

The boundary consistency measurement of a HFLPR is the measurement based on the endpoints of the HFLPR. Wu and Xu [62] employed the (equal) possibility distribution of the linguistic terms in a HFLTS in describing the preference degrees to obtain the expected value of the HFLTS.

Let $H=\left(H_{i j}\right)_{n \times n}$ be a HFLPR, then its corresponding expected LPR $E_{H}=\left(e h_{i j}\right)_{n \times n}$, where $e h_{i j}=\Delta\left(\alpha \Delta^{-1}\left(H_{i j}^{-}\right)+(1-\alpha) \Delta^{-1}\left(H_{i j}^{+}\right)\right)$with $0 \leq \alpha \leq 1$. In [62], to simplify the decision making process the value $\alpha=\frac{1}{2}$ is applied to obtain the values of $E_{H}$, which are constructed as the midpoint of the HFLPR values.

Definition 22 [62]. A HFLPR $H$ is called an additive consistent HFLPR, if and only if its expected LPR $E_{H}=\left(e h_{i j}\right)_{n \times n}$ satisfies

$$
\Delta^{-1}\left(e h_{i j}\right)=\Delta^{-1}\left(e h_{i k}\right)+\Delta^{-1}\left(e h_{k j}\right)-\frac{g}{2} \text { for all } i, j, k=1,2, \ldots, n
$$

With Eq. (37), an additive consistent LPR $C_{H}=\left(c h_{i j}\right)_{n \times n}$ can be obtained from $E_{H}$, where

$$
\Delta^{-1}\left(c h_{i j}\right)=\frac{1}{n-2} \sum_{k=1 ; k \neq i \neq j}^{n}\left(\Delta^{-1}\left(e h_{i k}\right)+\Delta^{-1}\left(e h_{k j}\right)-g / 2\right)
$$

Then, the consistency measurement of the HFLPR by measuring the difference between the expected LPR and its consistent LPR is proposed.

Definition 23 [62]. Let $H, E_{H}$ and $C_{H}$ be defined as above. The boundary consistency index for $H$ is computed as follows,

$$
C I(H)=1-d\left(E_{H}, C_{H}\right)=1-\frac{1}{n(n-1)} \sum_{i, j=1 ; i \neq j}^{n} \frac{\left|\Delta^{-1}\left(c h_{i j}\right)-\Delta^{-1}\left(e h_{i j}\right)\right|}{g}
$$

Essentially, the consistency measurement of a HFLPR proposed in [62] is based on the boundary linguistic values of the HFLPR, and it resembles the boundary consistency of the IVRPR, which was argued before that it cannot comprehensively reflect the consistency status of the RPR.

(3) Average consistency of HFLPRs

Li et al. in [33] presented the average consistency of a HFLPR by measuring the 
consistency of all the LPRs, with Eq. (14), associated to the HFLPR.

Definition 24 [33]. Let $H$ be a HFLPR and $L \in N_{H}$ be the LPRs associated to $H$. The average consistency index (ACI) of $H$ is,

$$
A C I(L)=\frac{1}{\# N_{H}} \sum_{L \in N_{H}} C I(L)
$$

where $\# N_{H}$ is the number of LPRs in $N_{H}$, i.e., $\# N_{H}=\prod_{i=1}^{n} \prod_{j=i+1}^{n}\left(\# H_{i j}\right)$.

Based on $\beta$-normalization, $\mathrm{Zhu}$ and $\mathrm{Xu}$ [63] introduced a parameter to add linguistic terms to HFLTSs to obtain a normalized HFLPR, i.e. a HFLPR with equal number of all HFLTSs, and the consistency of the HFLPR is determined by its normalized HFLPR. Li et al. [33] showed the mechanism of the consistency measurement of HFLPRs proposed in [63], which reflects the approximate average consistency degree of HFLPRs.

As mentioned before, because the additive consistency measurements approaches for hesitant APRs and hesitant MPRs are similar to the ones described for HFLPRs, a brief summary is provided: Zhu and $\mathrm{Xu}$ [18] and Zhu [64] developed regression methods to measure the optimistic consistency of the hesitant APR by transforming the hesitant APR into a reduced APR with highest consistency level. Inspired by the works of Dong et al. [15] and Li et al. [33], Zhang et al. [65] proposed optimization-based models to obtain the best consistency, worst consistency and average consistency by measuring the associated APRs of the hesitant APR. Zhang et al. [66] proposed the concept of a normalized hesitant APR by adding some elements in the original hesitant APR, and then by measuring the distance between the normalized hesitant APR and its consistent one the average consistency of hesitant APR is obtained. Zhang and $\mathrm{Wu}$ [67] developed a regression method to measure the optimistic consistency of a hesitant MPR. In [68], a normalization method of hesitant MPR is proposed to measure its consistency.

\section{Consistency-driven decision making}

In this section, consistency-driven decision making and and information fusion methods with RPRs based on the application of additive consistency measurements are reviewed, with the following proposed classification based on different decision making problems. 


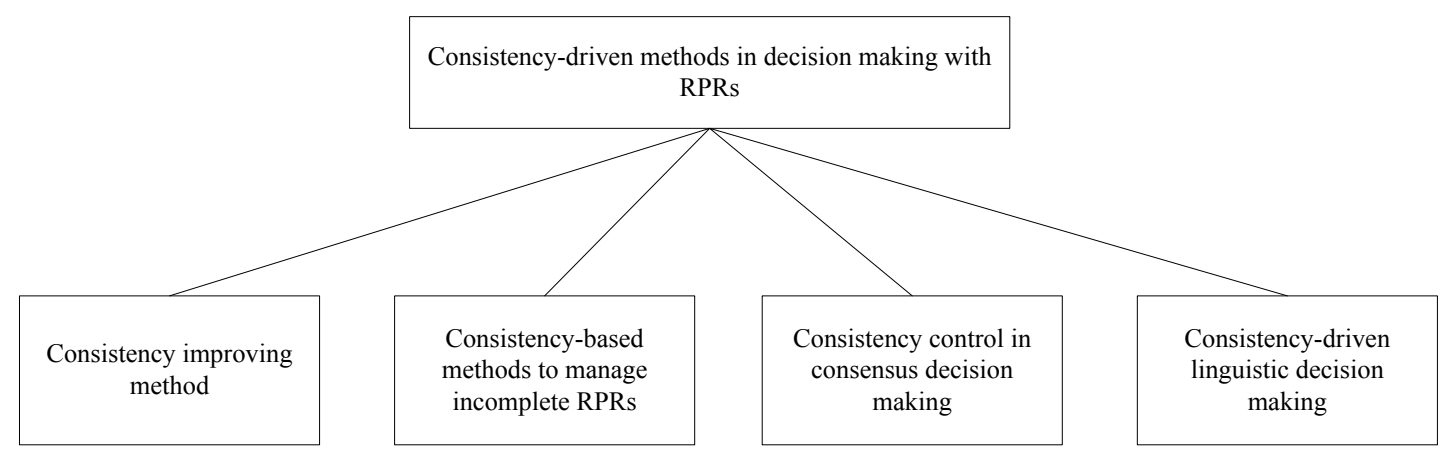

Fig. 2 A taxonomy of consistency-driven decision making and information fusion methods with RPRs

(1) Consistency improving method. When the RPRs provided by the decision makers are of unacceptable consistency, the consistency improving methods are used to help decision makers improve the consistency degree of inconsistent RPRs.

(2) Consistency-based methods to manage incomplete RPRs. In some situations, decision makers would not be able to efficiently express all the preference values and provide incomplete RPRs. Additive consistency property is often used as a tool to estimate the missing values in incomplete RPRs.

(3) Consistency control in consensus decision making. In group decision making, focusing on consensus reaching only could have an adverse effect on the consistency of RPRs. Thus, some investigations of individual consistency control within consensus model have been developed.

(4) Consistency-driven linguistic decision making, in which the consistency-driven methodology is used to set personalized numerical scale values for linguistic term sets under linguistic context, providing a new linguistic decision model to personalize individual semantics of linguistic terms.

Table 1 provides a summary of the consistency-driven methods used with the different types of RPRs.

Table 1. A summary of consistency-driven methods of RPRs

\begin{tabular}{|c|c|c|}
\hline $\begin{array}{c}\text { Consistency-driven decision } \\
\text { methods }\end{array}$ & Types of RPRs & References \\
\hline \multirow{3}{*}{$\begin{array}{c}\text { Consistency improving } \\
\text { method }\end{array}$} & APR & {$[22,69-73]$} \\
\cline { 2 - 3 } & MPR & {$[23,74-79]$} \\
\cline { 2 - 3 } & LPR & {$[10,11,43,80,81]$} \\
\cline { 2 - 3 } & IVAPR & {$[15]$} \\
\cline { 2 - 3 } & IVMPR & {$[54,58]$} \\
\cline { 2 - 3 } & IVLPR & {$[60]$} \\
\cline { 2 - 3 } & Hesitant APR & {$[18,63,65,82]$} \\
\cline { 2 - 3 } & Hesitant MPR & {$[68]$} \\
\hline
\end{tabular}




\begin{tabular}{|c|c|c|}
\hline & HFLPR & {$[61,63]$} \\
\hline Consistency-based methods & APR & {$[2,21,24,42,83-95]$} \\
\cline { 2 - 3 } to manage incomplete RPRs & MPR & {$[95-103]$} \\
\cline { 2 - 3 } & LPR & {$[42,99,104-106]$} \\
\cline { 2 - 3 } & IVAPR & {$[46,107,108]$} \\
\cline { 2 - 3 } & IVMPR & {$[109,110]$} \\
\cline { 2 - 3 } & IVLPR & {$[32]$} \\
\cline { 2 - 3 } consensus decision making & Hesitant APR & {$[65,111,112]$} \\
\cline { 2 - 3 } & APR & {$[2,22,94,113-117]$} \\
\cline { 2 - 3 } & MPR & {$[26,74,100,118]$} \\
\cline { 2 - 3 } & LPR & {$[27][104,119,120]$} \\
\cline { 2 - 3 } Consistency control in & HFLPR & {$[62]$} \\
\hline linguistic decision making & LPR and APR & {$[28,121,122]$} \\
\cline { 2 - 3 } & LPR and MPR & {$[123,124]$} \\
\cline { 2 - 3 } & LPR and IVAPR & {$[29,125,126]$} \\
\cline { 2 - 3 } & LPR and IVMPR & {$[127]$} \\
\cline { 2 - 3 } & LPR and HFLPR & \\
\hline
\end{tabular}

\subsection{Consistency improving method}

When the RPRs provided by decision makers are not of acceptable consistency, the consistency improving method is applied to repair the inconsistency of RPRs. The process of the consistency improving method is visualized in Fig. 3.

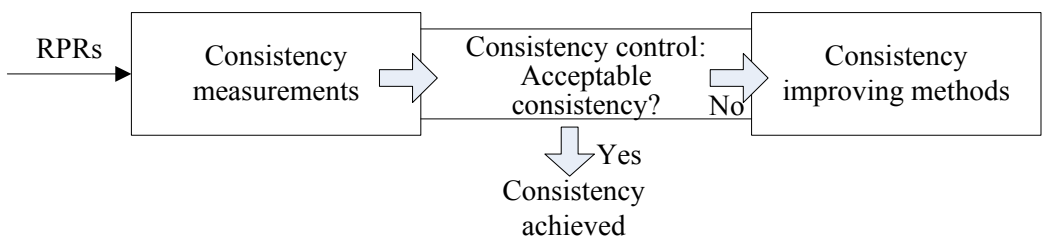

Fig. 3 General consistency improving process scheme

Two consistency improving approaches, the iterative approach and the optimization method, are often used in decision making with RPRs. In the following, we use APRs to illustrate the iterative method and the optimization-based method for consistency improving.

\subsubsection{Iterative approach}

Let $F=\left(f_{i j}\right)_{n \times n}$ be an APR with unacceptable consistency. An additive consistent APR $\bar{F}=\left(\overline{f_{i j}}\right)_{n \times n}$ can be constructed based on Eq. (2) $[22,69,70]$ via an optimization model [22], 


$$
\left\{\begin{array}{l}
\min \frac{1}{n^{2}} \sum_{i, j=1}^{n}\left|f_{i j}-\overline{f_{i j}}\right| \\
\text { s.t. } \\
\overline{f_{i j}} \geq 0, \quad i, j=1,2, \ldots, n \\
\overline{f_{i j}}+\overline{f_{j i}}=1, \quad i, j=1,2, \ldots, n \\
\overline{f_{i j}}+\overline{f_{j k}}-\overline{f_{i k}}=0.5, \quad i, j, k=1,2, \ldots, n
\end{array}\right.
$$

The main idea of the iterative approach for consistency improving is to help decision makers construct a new APR $F^{\prime}$ according to the consistent $\operatorname{APR} \bar{F}=\left(\overline{f_{i j}}\right)_{n \times n}$. When structuring the new APR $F^{\prime}$, it is suggested that $f_{i j}^{\prime} \in\left[\min \left\{f_{i j}, \overline{f_{i j}}\right\}, \max \left\{f_{i j}, \overline{f_{i j}}\right\}\right]$. This is indeed the basic idea of the iterative approaches for the consistency improving for the different types of RPRs. It has been first studied for MPR [23] in AHP and then further developed in [75]. In addition, the iterative approach has been used in the consistency improving with APR [69,71], MPR [74], LPR [10,11,128], interval-valued RPR [15,60] and hesitant RPR [18,63,68,82].

\subsubsection{Optimization-based method}

The main idea of the optimization-based model for dealing with inconsistent RPR is to find a suitable RPR with acceptable consistency, by preserving the original information as much as possible.

Let $F=\left(f_{i j}\right)_{n \times n}$ be an APR, and $F^{\prime}=\left(f_{i j}^{\prime}\right)_{n \times n}$ be the adjusted APR with acceptable consistency. In the consistency improving optimization model [22], the objective is to minimize the deviation degree between $F$ and $F^{\prime}$, i.e.

$$
\min \frac{1}{n(n-1)} \sum_{i, j=1 ; i \neq j}^{n}\left|f_{i j}-f_{i j}^{\prime}\right|
$$

Besides, the constructed APR $F^{\prime}$ should be of acceptable consistency, i.e.

$$
C I\left(F^{\prime}\right) \geq \overline{C I}
$$

Based on Eq. (3), Eq. (43) can be expressed as

$$
1-\frac{2}{3 n(n-1)(n-2)} \sum_{i, j, k=1 ; i \neq j \neq k}^{n}\left|f_{i j}^{\prime}+f_{j k}^{\prime}-f_{i k}^{\prime}-0.5\right| \geq \overline{C I}
$$

If $\overline{C I}=1$, then $F^{\prime}$ is set to be fully consistent and $f_{i j}^{\prime}+f_{j k}^{\prime}-f_{i k}^{\prime}=0.5$ is hold for all $i, j, k=1,2, \ldots, n$. 
With Eqs. (42) and (43), the following optimization-based model is proposed to improve the consistency of the APR,

$$
\left\{\begin{array}{l}
\min \frac{1}{n(n-1)} \sum_{i, j=1 ; i \neq j}^{n}\left|f_{i j}-f_{i j}^{\prime}\right| \\
\text { s.t. } \\
C I\left(F^{\prime}\right) \geq \overline{C I} \\
f_{i j}^{\prime}+f_{j i}^{\prime}=1, \quad i, j=1,2, \ldots, n
\end{array}\right.
$$

The basic idea of the optimization-based model has been used in the consistency improving with MPR [79], APR [22,72], LPR [10,11,80], IVPR [15] and HPR [65]. Additionally, $\mathrm{Xu}$ et al. [73,81,146] proposed an optimization-based model to deal with the additive inconsistencies for APR and LPR with the consideration of the ordinal consistency property.

\subsection{Consistency-based methods to manage incomplete RPRs}

Generally, there are two kinds of methods to estimate the missing values of incomplete preference relations based on the additive consistency measurements: the iterative procedure and the optimization-based procedure. We illustrate the iterative procedure and the optimization-based procedure to estimate the missing values of incomplete APRs.

\subsubsection{Iteration procedure}

The iteration approach used in incomplete APRs estimates missing preference values based on additive transitivity using all the possible intermediate alternatives for which preference values are known [21,91,92,129].

In an incomplete APR, given an unknown preference value $f_{i k}(i \neq k)$ the iterative approach starts by using intermediate alternatives $x_{j}$ and the additive transitivity property:

$$
f_{i k}^{j}=f_{i j}+f_{j k}-0.5
$$

Then, by averaging the values obtained from Eq. (45), the estimated value $f_{i k}$ is obtained,

$$
f_{i k}=\sum_{j=1, j \neq i \neq k}^{n} \frac{f_{i k}^{j}}{n-2}
$$

In each iteration, the unknown values are estimated using the known values provided by decision makers. The iterative procedure stops when all the unknown values are estimated. If the missing values cannot be all estimated from the iterative procedure then no preference 
values involving a particular alternative are known, and consequently the decision makers are required to provide more preference information.

An extension of the above iterative approach to deal with incomplete MPR, LPR and interval-valued RPR based on the consistency property is reviewed in [25]. Similar approaches are also discussed in [32,42,46,96,99,100,108].

\subsubsection{Optimization-based procedure}

The optimization-based procedure to estimate the missing values in an APR is constructed to minimize its additive inconsistency degree.

Let $F=\left(f_{i j}\right)_{n \times n}$ be an incomplete APR. Fedrizzi and Giove [24] proposed an optimization model by minimizing the global inconsistency of $F$,

$$
\rho=\sum_{i, j, k=1}^{n} L_{i j k}
$$

where $L_{i j k}=\left(f_{i j}+f_{j k}-f_{i k}-0.5\right)^{2}$ measures the inconsistency associated with alternatives $\left\{x_{i}, x_{j}, x_{k}\right\}$.

Zhang et al. [22] proposed a linear programming model to calculate the missing values of $F$ with the aim of maximizing the consistency degree of the completed APR $F^{\prime}=\left(f_{i j}^{\prime}\right)_{n \times n}$ with $f_{i j}^{\prime}=f_{i j}$ for non-null entries of $F$. The following linear programming model is proposed in [22],

$$
\left\{\begin{array}{l}
\max C I\left(F^{\prime}\right)=1-\frac{2}{3 n(n-1)(n-2)} \sum_{i, j, k=1 ; i \neq j \neq k}^{n}\left|f_{i j}^{\prime}+f_{j k}^{\prime}-f_{i k}^{\prime}-0.5\right| \\
\text { s.t. } \\
f_{i j}^{\prime} \geq 0 \quad i, j=1,2, \ldots, n \\
f_{i j}^{\prime}+f_{j i}^{\prime}=1 \quad i, j=1,2, \ldots, n \\
f_{i j}^{\prime}=f_{i j} \quad \text { for } f_{i j} \neq \text { null }
\end{array}\right.
$$

The optimization-based procedure to estimate the missing value by obtaining a complete RPR with acceptable consistency that has the minimal distance to the incomplete APR is also introduced in [83]. In addition, optimization approaches to estimate the priority vector so as to complete the APRs are studied in [84-87,90]. Furthermore, the optimization-based approaches are also used to manage incomplete MPR [101], incomplete LPR [104], incomplete interval-valued RPR [109,110] and incomplete hesitant RPR [111,112].

\subsection{Consistency control in consensus decision making}


The individual consistency and consensus measurements are basics in conducting the GDM with RPRs [27,113]. Consistency measurements ensure that the decision maker is being neither random nor illogical in his/her pairwise comparisons of alternatives, while consensus measures the degree of agreement among decision makers. Generally, the GDM involves a two-step procedure:

(1) Consistency improving process. This process aims to deal with RPRs that are of unacceptable consistency and a consistency improving method (refer to Section 3.1) is used to improve their consistency.

(2) Consensus reaching process [130-132]. Once all RPRs are of acceptable consistency, the consensus process is used to reach an acceptable consensus among all the decision makers following the application of some consensus rules.

\subsubsection{The approach with repeating consistency improving process}

In order to effectively manage individual consistency and consensus in the GDM with RPRs, Herrera et al. [27] and Chiclana et al. [113] initiated consensus frameworks to integrate individual consistency control in consensus process with repeating the consistency improving process to deal with LPRs and APRs, respectively.

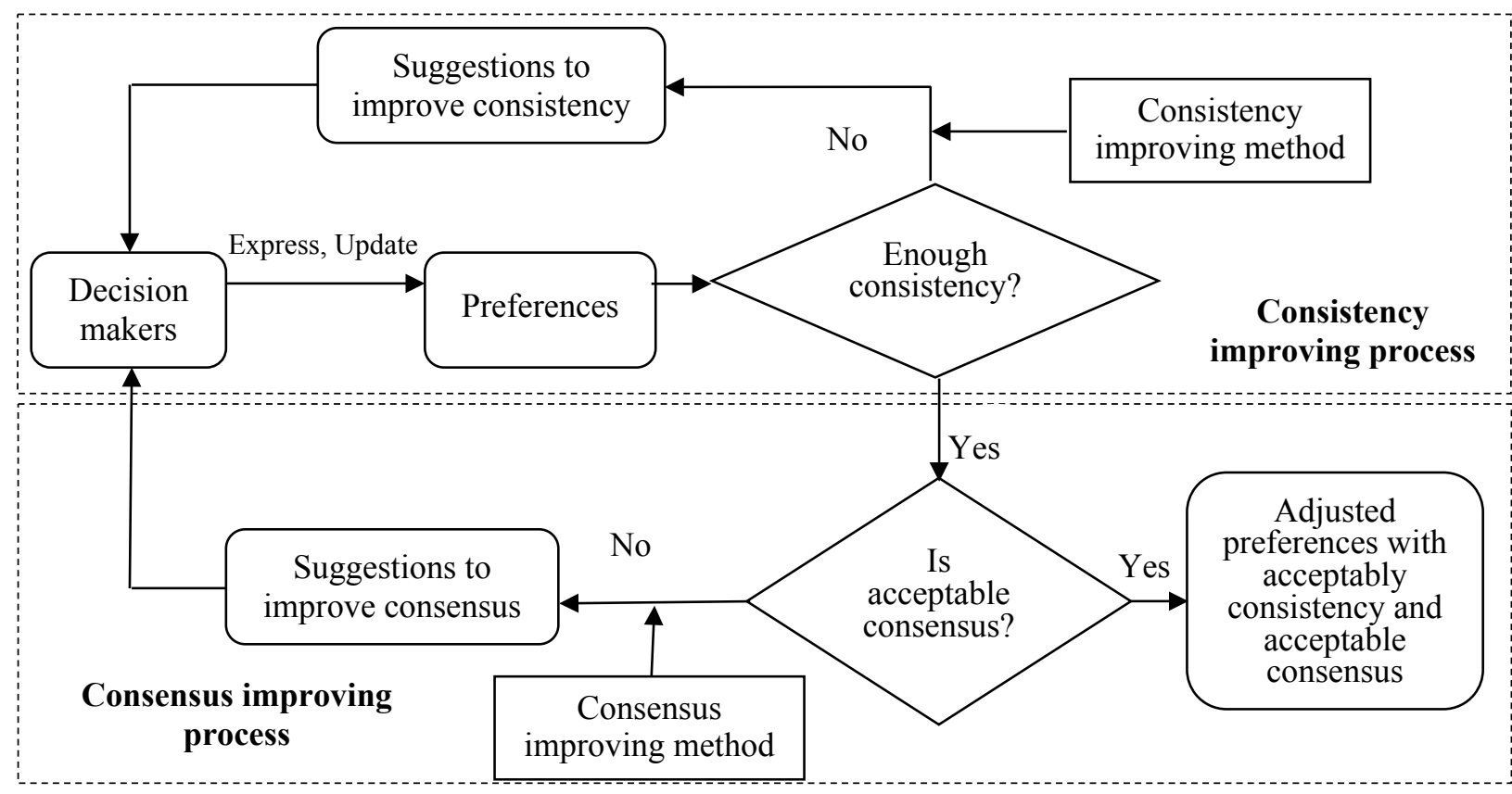

Fig.4 Consensus framework with individual consistency[113] [27]

Fig.4 provides the general framework of the consensus process with repeating the consistency improving process. In this framework, the individual consistency will often be destroyed by the consensus reaching process, which leads to repeat the consistency improving process until the adjusted RPRs with the acceptably consistency and acceptable consensus are 
obtained simultaneously. Consequently, the individual consistency is measured in each iteration before the consensus improving process is applied. Based on the preference relations provided by decision maker, first the consistency of the preference relations is measured. If the consistency is unacceptable, the consistency improving method is applied to provide suggestions to improve consistency. If the consistency is acceptable, then the consensus improving process is applied. If the RPRs are of unacceptable consensus, the consensus improving method is used to provide suggestions to improve consensus. Otherwise, output the adjusted preferences with acceptable consistency and acceptable consensus.

The consensus with individual consistency control for APRs, MPRs and LPRs has also been studied in $[2,100,104,106,115,119,120]$. Specifically, Herrera-Viedma et al. [2] presented a consensus process using consistency control with APRs, while Kamis et al. [116] proposed a consensus approach with personalized consistency control module with APRs to find the final consensus decision solution using a network structural equivalence clustering approach. Kian et al. [100] proposed an integrated Delphi-AHP consistency-checking consensus-building framework to deal with MPRs. Cabrerizo and Herrera-Viedma [106] provided a consensus model based on both consensus and consistency criteria to manage LPRs, while Zhao et al. [104] provided an iterative algorithm to deal with the consistency and the consensus in GDM with LPRs in which the consistency is repeatedly checked and improved.

\subsubsection{The approach without repeating consistency improving process}

It would be most efficient if individual consistency could be guaranteed in the consensus improving process without the repetitive application of consistency improving methods. Towards achieving this aim, Dong et al. [26] proposed an automatic consensus framework to address the GDM with MPRs by incorporating consistency and consensus measures into one phase. Based on this proposed framework, $\mathrm{Wu}$ and $\mathrm{Xu}[62,114]$ presented a consistency consensus based model with APRs and hesitant preference relation. Xia and Chen [74] developed a framework of the consistency and consensus methods for different types of classical RPRs based on Abelian linearly ordered group, while Zhang et al. [22] first proposed a linear optimization model for reaching consensus with consistency control with APRs.

Let $X=\left\{x_{1}, x_{2}, \ldots, x_{n}\right\}(n \geq 2)$ be a finite set of alternatives and $E=\left\{e_{1}, e_{2}, \ldots, e_{m}\right\}$ be a set of decision makers. Let $F^{k}=\left(f_{i j}^{k}\right)_{n \times n}(k=1,2, \ldots, m)$ be the individual APR provided by decision maker $e_{k}$, and $\overline{F^{k}}=\left(\overline{f_{i j}^{k}}\right)_{n \times n}(k=1,2, \ldots, m)$ the adjusted APR with acceptable 
consistency and consensus. Let $\overline{C I}$ and $\overline{C L}$ be the consistency and consensus threshold values, respectively. The following optimization model was proposed in [22]:

$$
\left\{\begin{array}{l}
\min \frac{1}{n^{2} m} \sum_{k=1}^{m} \sum_{i=1}^{n} \sum_{j=1}^{n}\left|f_{i j}^{k}-\overline{f_{i j}^{k}}\right| \\
\text { s.t. } \\
\frac{2}{3 n(n-1)(n-2)} \sum_{i, c=1 ; i \neq c}^{n} \sum_{j=1 ; j \neq i, c ;}^{n}\left|\overline{f_{i j}^{k}}+\overline{f_{j c}^{k}}-\overline{f_{i c}^{k}}-0.5\right| \leq 1-\overline{C I}, k=1,2, \ldots, m \\
\frac{2}{n m(m-1)(n-1)} \sum_{i=1}^{n} \sum_{j=1, j \neq i}^{n} \sum_{t \geq r}^{m} \sum_{r=1}^{m}\left(\overline{f_{i j}^{r}}-\overline{f_{i j}^{t}}\right) \leq 1-\overline{C L}
\end{array}\right.
$$

In the above studies, each individual RPR is always ensured to be of acceptable consistency in the achievement of the predefined consensus level. However, they are based on an automatic consensus process that does not consider decision makers' opinions during the consensus reaching process. Thus, recently Li et al. [117] proposed a consensus reaching algorithm with individual consistency control in GDM with APR that provides an optimized consensus rule to guarantee the individual consistency before applying the consensus process.

The consensus framework with individual consistency control without the repetitive of consistency improving approach is shown in Fig. 5.

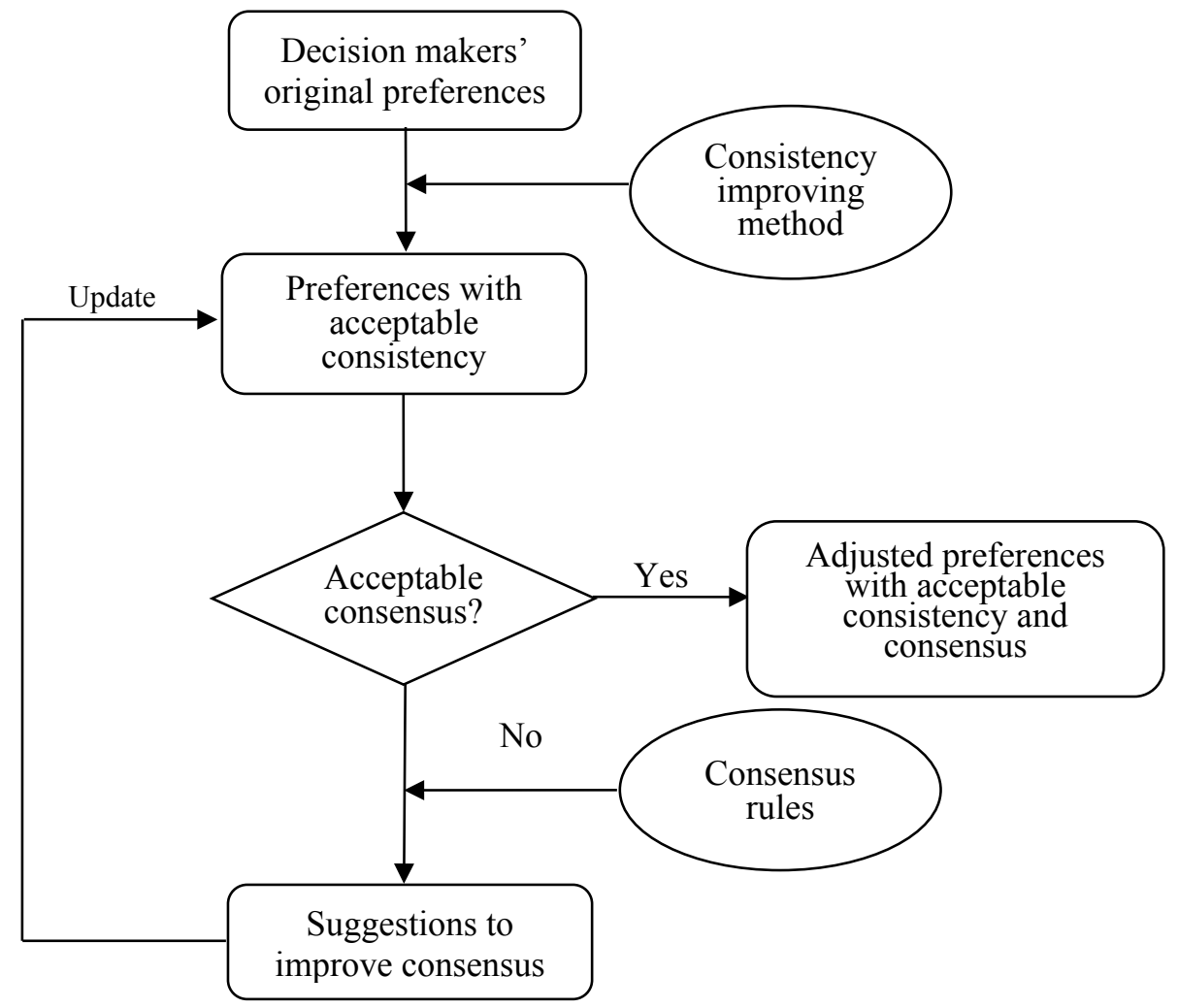

Fig. 5 The consensus framework with individual consistency control [117]

Firstly, the consistency improving method is applied to obtain preferences with 
acceptable consistency. The consistency improving process is only applied in the first round of the process, which avoids repeating the consistency improving process in the consensus reaching process. Secondly, based on the preference with acceptable consistency, the consensus degree is measured. If the group consensus is unacceptable, the consensus rules are applied to provide suggestions to improve consensus. Otherwise, output the adjusted preference with acceptable consistency and consensus.

Let $F^{c}=\left(f_{i j}^{c}\right)_{n \times n}$ be the collective APR obtained by aggregating $\left\{F^{1}, F^{2}, \ldots, F^{m}\right\}$. In [117], the basic idea of the consensus rule with individual consistency control is to obtain the adjustable range, $\left[l_{i j}^{k}, u_{i j}^{k}\right]$, for decision maker $e_{k}$ and pairwise $\left(x_{i}, x_{j}\right)$. When the value $f_{i j}^{k}$ is revised within $\left[l_{i j}^{k}, u_{i j}^{k}\right]$ in the consensus reaching process, the adjusted APR is of acceptably consistency and also the group consensus will improve. The consensus rule with individual consistency control is implemented via an optimization model. To guarantee the improvement of the consensus among decision makers, the adjustable range $\left[l_{i j}^{k}, u_{i j}^{k}\right]$ should be contained in the interval $\left[\min \left\{f_{i j}^{k}, f_{i j}^{c}\right\}, \max \left\{f_{i j}^{k}, f_{i j}^{c}\right\}\right]$, i.e., $\left[l_{i j}^{k}, u_{i j}^{k}\right] \subseteq\left[\min \left\{f_{i j}^{k}, f_{i j}^{c}\right\}, \max \left\{f_{i j}^{k}, f_{i j}^{c}\right\}\right]$. Denoting the set of APRs based on $\left[l_{i j}^{k}, u_{i j}^{k}\right]$ by $\varphi^{k}=\left\{A^{k}=\left(a_{i j}^{k}\right)_{n \times n} \mid a_{i j}^{k} \in\left[l_{i j}^{k}, u_{i j}^{k}\right], a_{i j}^{k}+a_{j i}^{k}=1, i, j=1,2, \ldots, n, k=1,2, \ldots, m\right\}$, the consistency of the adjusted APR is guaranteed if all $A^{k} \in \varphi^{k}$ are of acceptable consistency, i.e. $\min _{A^{k} \in \varphi^{k}} C I\left(A^{k}\right) \geq \overline{C I}$. Finally, the decision makers should have the maximum degree of freedom in revising the preference, so the width of $\left[l_{i j}^{k}, u_{i j}^{k}\right]$ is set to achieve $\max \sum_{i=1}^{n} \sum_{j=i+1}^{n}\left(u_{i j}^{k}-l_{i j}^{k}\right)$, i.e. the following optimization model to obtain the adjustable range $\left[l_{i j}^{k}, u_{i j}^{k}\right]$ is proposed [117]:

$$
\left\{\begin{array}{l}
\max \sum_{i=1}^{n} \sum_{j=i+1}^{n}\left(u_{i j}^{k}-l_{i j}^{k}\right) \\
\text { s.t. } \\
{\left[l_{i j}^{k}, u_{i j}^{k}\right] \subseteq\left[\min \left\{f_{i j}^{k}, f_{i j}^{c}\right\}, \max \left\{f_{i j}^{k}, f_{i j}^{c}\right\}\right]} \\
\min _{A^{k} \in \varphi^{k}} C I\left(A^{k}\right) \geq \overline{C I}
\end{array}\right.
$$

The solution of the proposed optimization model is obtained using an approximate algorithm with an adjustment parameter, which provides a good approximate performance[117]. Finally, based on the consensus rule with individual control, a consensus reaching process is designed to assist decision makers to reach a consensus in GDM with 
APRs.

\subsection{Consistency-driven linguistic decision making}

A proposed approach to computing with words decision making problems is the consistency-driven linguistic decision making procedures as proposed in $[29,125,126]$. In this framework, $S=\left\{s_{0}, s_{1}, \ldots, s_{g}\right\}$ represents a linguistic term set, which is usually modelled using the previously described 2-tuple linguistic model (see Section 2.2.3), which Dong et al. [133] extended by transforming linguistic terms into interval numerical scales.

Definition 25 [133]. Let $M=\left\{\left[A_{L}, A_{R}\right] \mid A_{L}, A_{R} \in[0,1], A_{L} \leq A_{R}\right\}$ be a set of interval values in $[0,1]$. The function INS:S $\rightarrow M$ is defined as an interval numerical scale of $S$, and $\operatorname{INS}\left(s_{i}\right)$ is called the interval numerical index of $s_{i}$. The interval numerical scale INS on $\bar{S}$ is defined by,

$\operatorname{INS}\left(\left(s_{i}, \alpha\right)\right)=\left[A_{L}, A_{R}\right]$

where

$$
\begin{aligned}
& A_{L}=\left\{\begin{array}{l}
I N S_{L}\left(s_{i}\right)+\alpha \times\left(I N S_{L}\left(s_{i+1}\right)-I N S_{L}\left(s_{i}\right)\right), \alpha \geq 0 \\
I N S_{L}\left(s_{i}\right)+\alpha \times\left(I N S_{L}\left(s_{i}\right)-I N S_{L}\left(s_{i-1}\right)\right), \alpha<0
\end{array}\right. \\
& A_{R}=\left\{\begin{array}{l}
I N S_{R}\left(s_{i}\right)+\alpha \times\left(I N S_{R}\left(s_{i+1}\right)-I N S_{R}\left(s_{i}\right)\right), \alpha \geq 0 \\
I N S_{R}\left(s_{i}\right)+\alpha \times\left(I N S_{R}\left(s_{i}\right)-I N S_{R}\left(s_{i-1}\right)\right), \alpha<0
\end{array}\right.
\end{aligned}
$$

The basic idea of the consistency-driven linguistic decision making method is to set personalized numerical value for linguistic term sets based on the consistency property. It is based on a natural premise regarding the consistency of LPRs.

Premise 1: If LPRs provided by decision makers are of acceptable consistency, the corresponding transformed numerical preference relations by the established numerical scales are also consistent.

Optimization models are often constructed in the consistency-driven linguistic decision making to deal with the translation process in computing with words. By applying the consistency-driven optimization model, the linguistic values are transformed into single numerical values or interval numerical values. Fig. 6 shows the basic idea of the consistencydriven methodology. 


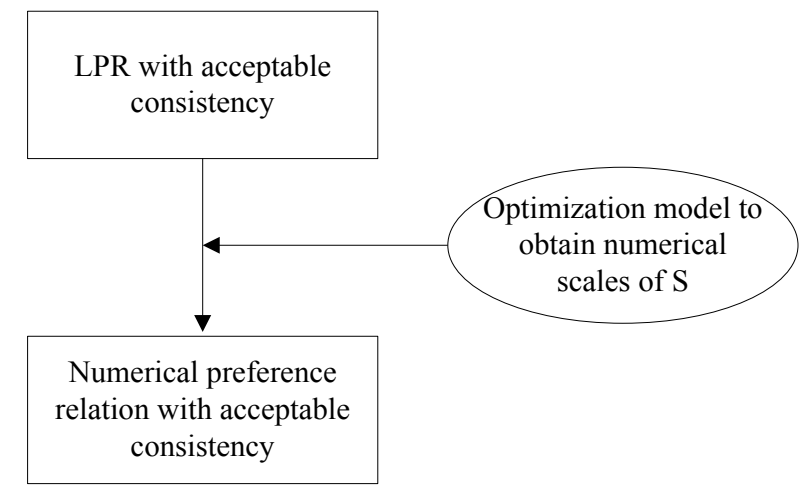

Fig. 6 Basic idea of the consistency-driven methodology[29,125,134]

Next, we illustrate the consistency-driven linguistic model to set the personalized interval numerical scales of linguistic terms to support linguistic decision making [29].

Let $E=\left\{e_{1}, e_{2}, \ldots, e_{m}\right\}$ be a set of decision makers, $S=\left\{s_{0}, s_{1}, \ldots, s_{g}\right\}$ be a linguistic term set. Let $I N S^{k}\left(s_{i}\right)=\left[A_{L}^{i, k}, A_{R}^{i, k}\right] \subseteq[0,1]$ be the interval numerical scale of $s_{i}$, associated with decision maker $e_{k}$. Let $L^{k}=\left(l_{i j}^{k}\right)_{n \times n}$, where $l_{i j}^{k} \in S$, be the LPR provided by $e_{k}$, and $\widetilde{V}^{k}=\left[v_{i j}^{k-}, v_{i j}^{k+}\right]_{n \times n}$ with $\left[v_{i j}^{k-}, v_{i j}^{k+}\right]=\operatorname{INS}\left(l_{i j}^{k}\right)$ be the transformed IVAPR. Based on Premise 1, the objective of the optimization-based model is to maximize the consistency of the transformed IVAPR $\widetilde{V}^{k}$, i.e., $\max C I\left(\widetilde{V}^{k}\right)$. INS $S^{k}$ must be ordered, so $A_{L}^{i, k}<A_{L}^{i+1, k}$ and $A_{R}^{i, k}<A_{R}^{i+1, k}$ for $i=0,1, \ldots, g-1$ are guaranteed. Let $b_{i}$ be the initial numerical index of $s_{i}$, then it is $0 \leq A_{L}^{i, k} \leq b_{i} \leq A_{R}^{i, k} \leq 1$ for $i=0,1, \ldots, g$. Then, the optimization-based model to obtain the personalized numerical scales of linguistic terms for different decision makers is [29]:

$$
\left\{\begin{array}{l}
\max C I\left(\widetilde{V}^{k}\right) \\
\text { s.t. } \\
A_{L}^{i, k}<A_{L}^{i+1, k} \quad i=0,1, \ldots, g-1 \\
A_{R}^{i, k}<A_{R}^{i+1, k} \quad i=0,1, \ldots, g-1 \\
0 \leq A_{L}^{i, k} \leq b_{i} \leq A_{R}^{i, k} \leq 1 \quad i=0,1, \ldots, g
\end{array}\right.
$$

where $C I\left(\widetilde{V}^{k}\right)$ is used to measure consistency degree in $\widetilde{V}^{k}$.

In [28], Dong et al. set the numerical scales of the linguistic term set by maximizing the consistency of the transformed numerical preference relation, while in [123] Dong et al. presented the individual numerical scale of LPR based on AHP consistency. In [125], a consistency-driven optimization-based model is provided to obtain the personalized interval 
numerical scales for linguistic terms with LPRs, which was used by Huang and Li [126] to propose a consensus decision making model with personalized individual semantics. Li et al. [122] proposed a consistency-driven approach to personalize individual semantics with LPRs in large-scale group decision making and further studied its use in consensus model. In [127], a consistency-driven methodology is proposed to deal with HFLPRs, from which the personalized numerical scales for linguistic terms in HFLTSs are obtained. In addition, the consistency-driven model has also been applied in the AHP $[124,135,136]$ and multi-criteria group decision making with LPRs [121].

\section{Summary, critical discussions and new directions}

In this section, we analyses the known consistency studies of RPRs and proposes new research directions for analysis.

\subsection{Summary and critical discussion}

RPRs are widely used to model knowledge and preferences in decision making. There exist various types of RPRs, which are classified in this review into classical RPRs, intervalvalued RPRs and hesitant RPRs. Classical RPRs, including APRs, MPRs and LPRs, are often used in decision making problems when decision makers choose to use a single numerical or linguistic value to express their preferences. Interval-valued RPRs, including IVAPRs, IVMPRs and IVLPRs, are usually used in complex decision making context where decision makers cannot use a single value to express their preferences and interval values are more appropriate. Hesitant RPRs, which includes hesitant APRs, hesitant MPRs and HFLPRs, are employed in decision making contexts where decision makers express hesitancy about their opinions in assessing the alternatives.

The additive consistency measurements for classical RPRs are classified into local and global methods, respectively. The consistency measurements of interval-valued RPRs are based on the classical RPRs measurement because an interval-valued RPR can be seen as a collection of classical RPRs [15]. Similarly, the consistency of hesitant RPRs is measured based on its associated classical RPRs. For interval-valued RPRs and hesitant RPRs, different methods have been proposed to reflect different aspects of consistency and to measure the consistency levels: the optimistic consistency, the pessimistic consistency, the boundary consistency and the average consistency.

Consistency-driven decision making methods are classified into four types: the consistency improving method, the consistency-based method to manage incomplete RPRs, 
the consistency control in consensus decision making and the consistency-driven linguistic decision making. They all are based on the use of the consistency measurements with different kinds of RPRs in decision making.

There are two different approaches in the consistency improving method: the iterative approach and the optimization-based approach. The iterative approach constructs the adjusted RPR by changing the original preferences toward the consistent preferences, while the optimization-based approach aims to find a new RPR with acceptable consistency that preserves the original information as much as possible. In the consistency-based method to manage incomplete RPRs, both the optimization-based procedure and the iterative procedure estimate the unknown values using the known preference values by maximizing consistency level. For the consistency control in consensus decision problem, the adjusted RPRs fulfill the requirements that the group consensus is not only improved, but also the required consistency is achieved. The consistency-driven linguistic decision making constructs consistency-driven linguistic decision method to personalize individual semantics and it can be used to deal with the fact that words mean different things for different people to support linguistic decision making.

Although various types of RPRs were defined and lots of studies were presented to analysis their consistency measurements and consistency-driven decision making, there are still some shortcomings that must be highlighted:

(1) Although many different types of RPRs have been reported in the existing studies, the basic ideas for the consistency measurements and consistency-driven decision methods for the different types of RPRs are similar, which leads to lots of confusions in investigating consistency issues in decision making.

(2) The setting of the consistency threshold value is an important issue that indicates whether the consistency level of an RPR can be considered acceptable. However, it is difficult to establish an appropriate consistency threshold value. Particularly, decision makers often have individual differences in judging whether the consistency level of a RPR is acceptable [11].

(3) Developed consistency indexes are usually numerical ones, but it seems natural to expect that consistency indexes might have a qualitative nature. Thus, developing linguistic consistency indexes to show consistency status of RPRs is necessary.

(4) The consistency-driven decision making methods are all based on natural assumptions, and are performing very well based on the use of the consistency measurements with RPRs. But, there is a lack of simulation experiments to justify the assumptions used in 
the consistency-driven decision making.

(5) There is a large number of theoretical studies regarding the consistency measurements and the consistency-driven decision making methods but relatively few works on applications of these consistency approaches.

\subsection{New directions for analysis}

Thus, the following directions should be considered for research developing:

(1) Investigate further methods regarding the appropriate setting of consistency threshold values. Particularly, it would be very interesting to develop a personalized consistency threshold setting method. Meanwhile, we should develop linguistic consistency indexes that are convenient for decision makers to judge the consistency level of an RPR linguistically.

(2) A number of simulation experiments and real-life applications are needed to further justify the validity and feasibility of the different consistency measurements and the consistency-driven decision making methods.

(3) From the perspective of self-confidence levels, incomplete RPRs have two selfconfidence levels: complete and incomplete, and thus are a special case of the preference relations with multiple self-confidence levels [137]. It seems timely to study the consistency measurements and consistency-driven decision making methods for self-confidence preference relations, and to discuss the influence of the self-confidence levels in consistency measurements and consistency-driven decision making.

(4) With the development of information and network technology, social network [138] and opinion dynamics [139] are often involved in decision making and particularly in largescale decision making problems. It would be interesting to study the consistency issues of RPRs under social network and preference evolution contexts.

(5) A unified framework is necessary to connect the different types of RPRs, and to analyze their respective consistency measurements and consistency-driven decision making methods, avoiding repetitive research studies with similar ideas. Particularly, recently several researchers [142-144] studied the consistency issue via an axiomatic design, which seems to be a promising research direction to develop for consistency-driven decision making methods.

(6) There exist different ways to define consistency of RPRs (e.g., multiplicative consistency[145] and order consistency [146]). It would be useful to analysis the differences among different consistency definitions and to study the influence of different consistency definitions on decision outcomes. 


\section{Conclusion}

Additive consistency is widely used in decision making with RPRs. This paper reviews and analyzes the state-of-the-art of RPRs in decision making from the perspective of the additive consistency measurements and the consistency-driven decision making methods. Specifically, we first review the additive consistency measurements for different types of RPRs. We show that the additive consistency measurements for classical RPRs are classified into local and global methods, respectively. Moreover, the interval-valued RPRs and hesitant RPRs can be seen as a collection of classical RPRs, and their consistency measurements are based on that of the classical RPRs. Various methods reflecting different aspects of consistency have been proposed to measure the consistency levels of interval-valued RPRs and the hesitant RPRs, such as the optimistic consistency, the pessimistic consistency, the boundary consistency and the average consistency. The consistency-driven decision making methods are also reviewed and classified into four types: the consistency improving method, the consistency-based method to manage incomplete RPRs, the consistency control in consensus decision making, and the consistency-driven linguistic decision making. Finally, we critically summarize consistency issues of RPRs, and note new directions that are timely for future research.

\section{Acknowledgments}

We would like to acknowledge the financial support of the grants (Nos. 71871149 and 71571124) from NSF of China, the grants (Nos. sksyl201705 and 2018hhs-58) from Sichuan University, and the grant TIN2016-75850-R supported by the Spanish Ministry of Economy and Competitiveness with FEDER funds.

\section{References}

[1] T. Tanino, Fuzzy preference relations in group decision making, in: Non-Conventional Prefer. Relations Decis. Mak., Springer, 1988: pp. 54-71.

[2] E. Herrera-Viedma, S. Alonso, F. Chiclana, F. Herrera, A consensus model for group decision making with incomplete fuzzy preference relations, IEEE Trans. Fuzzy Syst. 15 (2007) 863-877.

[3] B. De Baets, H. De Meyer, B. De Schuymer, Cyclic evaluation of transitivity of reciprocal relations, Soc. Choice Welfare. 26 (2006) 217-238.

[4] J.C. Fodor, M.R. Roubens, Fuzzy preference modelling and multicriteria decision support, Springer Science \& Business Media, 1994.

[5] E. Herrera-Viedma, F. Herrera, F. Chiclana, M. Luque, Some issues on consistency of fuzzy preference relations, European Journal of Operational Research, Eur. J. Oper. Res. 154 (2004) 98-109. 
[6] G. Crawford, C. Williams, A note on the analyis of subjective judgment matrices, J. Math. Psychol. 29 (1985) 387-405.

[7] T.L. Saaty, The Analytic Hierarchy Process, New York McGraw Hill. Int. 48 (1980) 9-26.

[8] Z.-P. Fan, J. Ma, Y.-P. Jiang, Y.-H. Sun, L. Ma, A goal programming approach to group decision making based on multiplicative preference relations and fuzzy preference relations, Eur. J. Oper. Res. 174 (2006) 311-321.

[9] B. Srdjevic, Combining different prioritization methods in the analytic hierarchy process synthesis, Comput. Oper. Res. 32 (2005) 1897-1919.

[10] Y. Dong, Y. Xu, H. Li, On consistency measures of linguistic preference relations, Eur. J. Oper. Res. 189 (2008) 430-444.

[11] Y. Dong, W.C. Hong, Y. Xu, Measuring consistency of linguistic preference relations: A 2-tuple linguistic approach, Soft Comput. 17 (2013) 2117-2130.

[12] F. Herrera, E. Herrera-Viedma, J.L. Verdegay, Preference degrees over linguistic preference relations in decision making, BADANIA OPERACYJNE I Decyz. (1995) 37-48.

[13] F. Herrera, E. Herrera-Viedma, J.L. Verdegay, A sequential selection process in group decision making with a linguistic assessment approach, Inf. Sci. (Ny). 85 (1995) 223-239.

[14] Z. Xu, J. Chen, Some models for deriving the priority weights from interval fuzzy preference relations, Eur. J. Oper. Res. 184 (2008) 266-280.

[15] Y. Dong, C.C. Li, F. Chiclana, E. Herrera-Viedma, Average-case consistency measurement and analysis of interval-valued reciprocal preference relations, Knowledge-Based Syst. 114 (2016) 108-117.

[16] Z. Xu, Consistency of interval fuzzy preference relations in group decision making, Appl. Soft Comput. 11 (2011) 3898-3909.

[17] R.M. Rodriguez, L. Martinez, F. Herrera, Hesitant fuzzy linguistic term sets for decision making, IEEE Trans. Fuzzy Syst. 20 (2012) 109-119.

[18] MM. Xia, ZS. Xu, Managing hesitant information in GDM problems under fuzzy and multiplicative preference relations, International Journal of Uncertainty, Fuzziness and Knowledge-Based Systems 21(6)(2013) 865-897.

[19] D. Dubois, H.P. Prade, Fuzzy Sets and Systems: Theory and Applications, Academic press, 1980.

[20] T. Tanino, Fuzzy preference orderings in group decision making, Fuzzy Sets Syst. 12 (1984) 117-131.

[21] E. Herrera-Viedma, F. Chiclana, F. Herrera, S. Alonso, Group decision-making model with incomplete fuzzy preference relations based on additive consistency, Syst. Man, Cybern. Part B Cybern. IEEE Trans. 37 (2007) 176-189.

[22] G. Zhang, Y. Dong, Y. Xu, Linear optimization modeling of consistency issues in group decision making based on fuzzy preference relations, Expert Syst. Appl. 39 (2012) 2415-2420.

[23] E. Blankmeyer, Approaches to consistency adjustment, J. Optim. Theory Appl. 54 (1987) 479-488.

[24] M. Fedrizzi, S. Giove, Incomplete pairwise comparison and consistency optimization, Eur. J. Oper. Res. 183 (2007) 303-313.

[25] R. Ureña, F. Chiclana, J.A. Morente-Molinera, E. Herrera-Viedma, Managing incomplete preference relations in decision making: a review and future trends, Inf. Sci. (Ny). 302 (2015) 14-32.

[26] Y. Dong, G. Zhang, W.C. Hong, Y. Xu, Consensus models for AHP group decision making under row geometric mean prioritization method, Decis. Support Syst. 49 (2010) 281-289.

[27] F. Herrera, E. Herrera-Viedma, J.L. Verdegay, A rational consensus model in group decision making using linguistic, Fuzzy Sets Syst. 88 (1997) 31--49. 
[28] Y. Dong, Y. Xu, S. Yu, Computing the numerical scale of the linguistic term set for the 2-tuple fuzzy linguistic representation model, IEEE Trans. Fuzzy Syst. 17 (2009) 1366-1378.

[29] Y. Dong, E. Herrera-Viedma, Consistency-driven automatic methodology to set interval numerical scales of 2-Tuple linguistic term sets and its use in the linguistic GDM with preference relation, IEEE Trans. Cybern. 45 (2015) 780-792.

[30] T.L. Saaty, Decision making with the AHP, Why is the principal eigenvector necessary, Eur. J. Oper. Res. 145 (2003) 85-91.

[31] Y.M. Wang, J.B. Yang, D.L. Xu, Interval weight generation approaches based on consistency test and interval comparison matrices, Appl. Math. Comput. 167 (2005) 252-273.

[32] Z. Zhang, C. Guo, Consistency-based algorithms to estimate missing elements for uncertain 2-tuple linguistic preference relations, Int. J. Comput. Intell. Syst. 7 (2014) 924-936.

[33] C.C. Li, R.M. Rodríguez, L. Martínez, Y. Dong, F. Herrera, Consistency of hesitant fuzzy linguistic preference relations: An interval consistency index, Inf. Sci. (Ny). 432 (2018) 347-361.

[34] F. Chiclana, F. Herrera, E. Herrera-Viedma, A note on the internal consistency of various preference representations, Fuzzy Sets Syst. 131 (2002) 75-78.

[35] J. Aguaron, J.M. Moreno-Jiménez, The geometric consistency index: Approximated thresholds, Eur. J. Oper. Res. 147 (2003) 137-145.

[36] L.A. Zadeh, The concept of a linguistic variable and its application to approximate reasoning - I, Inf. Sci. (Ny). 8 (1975) 199-249.

[37] F. Herrera, S. Alonso, F. Chiclana, E. Herrera-Viedma, Computing with words in decision making: foundations, trends and prospects, Fuzzy Optim. Decis. Mak. 8 (2009) 337-364.

[38] L. Martinez, D. Ruan, F. Herrera, Computing with words in decision support systems: an overview on models and applications, Int. J. Comput. Intell. Syst. 3 (2010) 382-395.

[39] L. Martínez, R.M. Rodriguez, F. Herrera, The 2-tuple Linguistic Model: Computing with Words in Decision Making, Springer, 2015.

[40] J. Mendel, D. Wu, Perceptual computing: Aiding people in making subjective judgments, John Wiley \& Sons, 2010.

[41] F. Herrera, L. Martínez, A 2-tuple fuzzy linguistic representation model for computing with words, IEEE Trans. Fuzzy Syst. 8 (2000) 746-752.

[42] S. Alonso, F. Chiclana, F. Herrera, E. Herrera-Viedma, J. Alcalá-Fdez, C. Porcel, A consistency-based procedure to estimate missing pairwise preference values, Int. J. Intell. Syst. 23 (2008) 155-175.

[43] F. Jin, Z. Ni, H. Chen, Y. Li, Approaches to decision making with linguistic preference relations based on additive consistency, Appl. Soft Comput. 49 (2016) 71-80.

[44] X. Chen, H. Zhang, Y. Dong, The fusion process with heterogeneous preference structures in group decision making: A survey, Inf. Fusion. 24 (2015) 72-83.

[45] F. Liu, W.-G. Zhang, J.-H. Fu, A new method of obtaining the priority weights from an interval fuzzy preference relation, Inf. Sci. (Ny). 185 (2012) 32-42.

[46] Z.J. Wang, A note on "incomplete interval fuzzy preference relations and their applications", Comput. Ind. Eng. 77 (2014) 65-69.

[47] J. Krejčí, On additive consistency of interval fuzzy preference relations, Comput. Ind. Eng. 107 (2017) $128-140$.

[48] F. Liu, Y.N. Peng, Q. Yu, H. Zhao, A decision-making model based on interval additive reciprocal matrices with additive approximation-consistency, Inf. Sci. (Ny). 422 (2018) 161-176. 
[49] Z.J. Wang, K.W. Li, Goal programming approaches to deriving interval weights based on interval fuzzy preference relations, Inf. Sci. (Ny). 193 (2012) 180-198.

[50] Y. Xu, K.W. Li, H. Wang, Incomplete interval fuzzy preference relations and their applications, Comput. Ind. Eng. 67 (2014) 93-103.

[51] Q. Yue, Z. Fan, L. Shi, New approach to determine the priorities from interval fuzzy preference relations, J. Syst. Eng. Electron. 22 (2011) 267-273.

[52] P. de Jong, A statistical approach to Saaty's scaling method for priorities, J. Math. Psychol. 28 (1984) 467-478.

[53] L. Wasseman, All of Statistics, 2004.

[54] Y. Dong, X. Chen, C.C. Li, W.-C. Hong, Y. Xu, Consistency issues of interval pairwise comparison matrices, Soft Comput. 19 (2015) 2321-2335.

[55] Y.M. Wang, J.B. Yang, D.L. Xu, A two-stage logarithmic goal programming method for generating weights from interval comparison matrices, Fuzzy Sets Syst. 152 (2005) 475-498.

[56] R. Islam, M.P. Biswal, S.S. Alam, Preference programming and inconsistent interval judgments, Eur. J. Oper. Res. 97 (1997) 53-62.

[57] F. Liu, Acceptable consistency analysis of interval reciprocal comparison matrices, Fuzzy Sets Syst. 160 (2009) 2686-2700.

[58] H. Zhang, A consistency model for group decision making problems with interval multiplicative preference relations, Appl. Soft Comput. 34 (2015) 60-71.

[59] F. Liu, W. Pedrycz, W.G. Zhang, Limited rationality and its quantification through the interval number judgments with permutations, IEEE Trans. Cybern. 47 (2017) 4025-4037.

[60] S. Yao, J. Hu, A minimum deviation approach for improving the consistency of uncertain 2-tuple linguistic preference relations, Comput. Ind. Eng. 117 (2018) 181-190.

[61] X. Feng, L. Zhang, C. Wei, The consistency measures and priority weights of hesitant fuzzy linguistic preference relations, Appl. Soft Comput. 65 (2018) 79-90.

[62] Z. Wu, J. Xu, Managing consistency and consensus in group decision making with hesitant fuzzy linguistic preference relations, Omega. 65 (2016) 28-40.

[63] B. Zhu, Z. S. Xu, Consistency measures for hesitant fuzzy linguistic preference relations, IEEE Trans. Fuzzy Syst. 22 (2014) 35-45.

[64] B. Zhu, Studies on consistency measure of hesitant fuzzy preference relations, Procedia Comput. Sci. 17 (2013) 457-464.

[65] Z. Zhang, X. Kou, Q. Dong, Additive consistency analysis and improvement for hesitant fuzzy preference relations, Expert Syst. Appl. 98 (2018) 118-128.

[66] Z. Zhang, C. Wang, X. Tian, A consensus model for group decision making with hesitant fuzzy information, Knowledge-Based Syst. 86 (2015) 77-101.

[67] W.C. Zhang Z, Deriving the priority weights from hesitant multiplicative preference relations in group decision making, Appl. Soft Comput. 25 (2014) 107-117.

[68] Z. Zhang, C. Wu, A decision support model for group decision making with hesitant multiplicative preference relations, Inf. Sci. (Ny). 282 (2014) 107-117.

[69] J. Ma, Z.P. Fan, Y.P. Jiang, J.Y. Mao, L. Ma, A method for repairing the inconsistency of fuzzy preference relations, Fuzzy Sets Syst. 157 (2006) 20-33.

[70] A.A. Al Salem, A. Awasthi, Investigating rank reversal in reciprocal fuzzy preference relation based on additive consistency: Causes and solutions, Comput. Ind. Eng. 115 (2018) 573-581. 
[71] M. Xia, Z. Xu, J. Chen, Algorithms for improving consistency of reciprocal preference relations, Fuzzy Sets Syst. 216 (2013) 108-133.

[72] Y. Xu, X. Liu, H. Wang, The additive consistency measure of fuzzy reciprocal preference relations, Int. J. Mach. Learn. Cybern. 9 (2018) 1141-1152.

[73] Y. Xu, F. Herrera, H. Wang, A distance-based framework to deal with ordinal and additive inconsistencies for fuzzy reciprocal preference relations, Inf. Sci. (Ny). 328 (2016) 189-205.

[74] M. Xia, J. Chen, Consistency and consensus improving methods for pairwise comparison matrices based on Abelian linearly ordered group, Fuzzy Sets Syst. 266 (2015) 1-32.

[75] Z. Xu, C. Wei, A consistency improving method in the analytic hierarchy process, Eur. J. Oper. Res. 116 (1999) 443-449.

[76] J. Benítez, X. Delgado-Galván, J. Izquierdo, R. Pérez-García, Consistent matrices and consistency improvement in decision-making processes, in: Proc. 7th Int. Conf. Eng. Comput. Technol., 2010.

[77] D. Cao, L.C. Leung, J.S. Law, Modifying inconsistent comparison matrix in analytic hierarchy process: A heuristic approach, Decis. Support Syst. 44 (2008) 944-953.

[78] W.Y. Ma, A practical approach to modifying pair wise comparison matrices and two criteria of modificatory effectiveness, J. Syst. Sci. Syst. Eng. 3 (1994) 334-338.

[79] J.S. Finan, W.J. Hurley, The analytic hierarchy process: does adjusting a pairwise comparison matrix to improve the consistency ratio help?, Comput. Oper. Res. 24 (1997) 749-755.

[80] Y. Dong, C.C. Li, F. Herrera, An optimization-based approach to adjusting unbalanced linguistic preference relations to obtain a required consistency level, Inf. Sci. (Ny). 292 (2015) 27-38.

[81] Y. Xu, D. Wu, H. Wang, A Gower plot-based approach to ascertain and adjust the ordinal and additive inconsistencies for fuzzy linguistic preference relations, Int. J. Fuzzy Syst. 19 (2017) 2003-2019.

[82] Z. Zhang, C. Wang, X. Tian, A decision support model for group decision making with hesitant fuzzy preference relations, Knowledge-Based Syst. 86 (2015) 77-101.

[83] F. Meng, X. Chen, A new method for group decision making with incomplete fuzzy preference relations, Knowledge-Based Syst. 73 (2015) 111-123.

[84] Z.W. Gong, Least-square method to priority of the fuzzy preference relations with incomplete information, Int. J. Approx. Reason. 47 (2008) 258-264.

[85] Z.S. Xu, J. Chen, Group decision-making procedure based on incomplete reciprocal relations, Soft Comput. 12 (2008) 515-521.

[86] Y. Xu, R. Patnayakuni, H. Wang, Logarithmic least squares method to priority for group decision making with incomplete fuzzy preference relations, Appl. Math. Model. 37 (2013) 2139-2152.

[87] Z.S. Xu, Goal programming models for obtaining the priority vector of incomplete fuzzy preference relation, Int. J. Approx. Reason. 36 (2004) 261-270.

[88] R. Ureña, F. Chiclana, H. Fujita, E. Herrera-Viedma, Confidence-consistency driven group decision making approach with incomplete reciprocal intuitionistic preference relations, Knowledge-Based Syst. 89 (2015) 86-96.

[89] Y. Zhang, H. Ma, Q. Li, B. Liu, J. Liu, Conditions of two methods for estimating missing preference information, Inf. Sci. (Ny). 279 (2014) 186-198.

[90] Y.J. Xu, Q.L. Da, L.H. Liu, Normalizing rank aggregation method for priority of a fuzzy preference relation and its effectiveness, Int. J. Approx. Reason. 50 (2009) 1287-1297.

[91] S.-M. Chen, T.-E. Lin, L.-W. Lee, Group decision making using incomplete fuzzy preference relations based on the additive consistency and the order consistency, Inf. Sci. (Ny). 259 (2014) 1-15. 
[92] L.-W. Lee, Group decision making using incomplete fuzzy preference relations based on the additive consistency and the order consistency, Expert Syst. Appl. 39 (2012) 11666-11676.

[93] S. Alonso, F. Chiclana, F. Herrera, E. Herreraviedma, A learning procedure to estimate missing values in fuzzy preference relations based on additive consistency, Model. Decis. Artif. Intell. 3131 (2004) 227238.

[94] S. Alonso, E. Herrera-Viedma, F. Chiclana, F. Herrera, Individual and social strategies to deal with ignorance situations in multi-person decision making, Int. J. Inf. Technol. Decis. Mak. 08 (2009) 313333.

[95] R. Ureña, F. Chiclana, S. Alonso, J.A. Morente-Molinera, E. Herrera-Viedma, On incomplete fuzzy and multiplicative preference relations in multi-person decision making, Procedia Comput. Sci. 31 (2014) 793-801.

[96] H. Zhang, Group decision making based on incomplete multiplicative and fuzzy preference relations, Appl. Soft Comput. 48 (2016) 735-744.

[97] S. Bozóki, J. Fülöp, L. Rónyai, On optimal completions of incomplete pairwise comparison matrices, Math. Comput. Model. 52 (2009) 318-333.

[98] V. Mattila, K. Virtanen, Ranking and selection for multiple performance measures using incomplete preference information, Eur. J. Oper. Res. 242 (2015) 568-579.

[99] F. Meng, X. Chen, An approach to incomplete multiplicative preference relations and its application in group decision making, Inf. Sci. (Ny). 309 (2015) 119-137.

[100] M.R.E. Kian, M. Sun, F. Bosché, A consistency-checking consensus-building method to assess complexity of energy megaprojects, Procedia - Soc. Behav. Sci. 226 (2016) 43-50.

[101] E. Dopazo, M. Ruiz-Tagle, A parametric GP model dealing with incomplete information for group decision-making, Appl. Math. Comput. 218 (2011) 514-519.

[102] K. Chen, G. Kou, J.M. Tarn, Y. Song, Bridging the gap between missing and inconsistent values in eliciting preference from pairwise comparison matrices, Ann. Oper. Res. 235 (2015) 155-175.

[103] F.J. Carmone Jr, A. Kara, S.H. Zanakis, A Monte Carlo investigation of incomplete pairwise comparison matrices in AHP, Eur. J. Oper. Res. 102 (1997) 538-553.

[104] M. Zhao, X.Y Ma, D.W Wei, A method considering and adjusting individual consistency and group consensus for group decision making with incomplete linguistic preference relations, Appl. Soft Comput. 54 (2017) 322-346.

[105] H. Wang, Z. Xu, Interactive algorithms for improving incomplete linguistic preference relations based on consistency measures, Appl. Soft Comput. 42 (2016) 66-79.

[106] F.J. Cabrerizo, I.J. Pérez, E. Herrera-Viedma, Managing the consensus in group decision making in an unbalanced fuzzy linguistic context with incomplete information, Knowledge-Based Syst. 23 (2010) 169-181.

[107] Y. J. Xu, K.W. Li, H.M. Wang, Incomplete interval fuzzy preference relations and their applications, Comput. Ind. Eng. 67 (2014) 93-103.

[108] A. Khalid, I. Beg, Incomplete interval valued fuzzy preference relations, Inf. Sci. (Ny). 348 (2016) 1524.

[109] F. Liu, W.-G. Zhang, Z.-X. Wang, A goal programming model for incomplete interval multiplicative preference relations and its application in group decision-making, Eur. J. Oper. Res. 218 (2012) 747-754.

[110] Z.-J. Wang, A note on "A goal programming model for incomplete interval multiplicative preference relations and its application in group decision-making”, Eur. J. Oper. Res. 247 (2015) 867-871. 
[111] Y. Xu, L. Chen, R.M. Rodríguez, F. Herrera, H. Wang, Deriving the priority weights from incomplete hesitant fuzzy preference relations in group decision making, Knowledge-Based Syst. 99 (2016) 71-78.

[112] Z. Zhang, X. Kou, W. Yu, C. Guo, On priority weights and consistency for incomplete hesitant fuzzy preference relations, Knowledge-Based Syst. 143 (2018) 115-126.

[113] F. Chiclana, F. Mata, L. Martinez, E. Herrera-Viedma, S. Alonso, Integration of a consistency control module within a consensus model, Int. J. Uncertainty, Fuzziness Knowledge-Based Syst. 16 (2008) 3553.

[114] Z. Wu, J. Xu, A concise consensus support model for group decision making with reciprocal preference relations based on deviation measures, Fuzzy Sets Syst. 206 (2012) 58-73.

[115] M. Fedrizzi, M. Fedrizzi, R.A.M. Pereira, On the issue of consistency in dynamical consensual aggregation, in: Technol. Constr. Intell. Syst. 1, Springer, 2002: pp. 129-137.

[116] N.H. Kamis, F. Chiclana, J. Levesley, Geo-uninorm consistency control module for preference similarity network hierarchical clustering based consensus model, Knowledge-Based Syst. (2018).

[117] C.C. Li, R.M. Rodriguez, L. Martinez, Y. Dong, F. Herrera, Consensus building with individual consistency control in group decision making, IEEE Trans. Fuzzy Syst. (2018), DOI: 10.1109/TFUZZ.2018.2856125.

[118] Z. Wu, J. Xu, A consistency and consensus based decision support model for group decision making with multiplicative preference relations, Decis. Support Syst. 52 (2012) 757-767.

[119] Y. Dong, C.-C. Li, Y. Xu, X. Gu, Consensus-based group decision making under multi-granular unbalanced 2-tuple linguistic preference relations, Group Decis. Negot. 24 (2015) 217-242.

[120] G. Zhang, Y. Dong, Y. Xu, Consistency and consensus measures for linguistic preference relations based on distribution assessments, Inf. Fusion. 17 (2014) 46-55.

[121] Y. Wang, Y. Dong, H. Zhang, Y. Gao, Personalized individual semantics based approach to MAGDM with the linguistic preference information on alternatives, Int. J. Comput. Intell. Syst. 11 (2018) 496-513.

[122] C.-C. Li, Y. Dong, F. Herrera, A consensus model for large-scale linguistic group decision making with a feedback recommendation based on clustered personalized individual semantics and opposing consensus groups, IEEE Trans. Fuzzy Syst. (2018), DOI: 10.1109/TFUZZ.2018.2857720.

[123] Y. Dong, W.C. Hong, Y. Xu, S. Yu, Numerical scales generated individually for analytic hierarchy process, Eur. J. Oper. Res. 229 (2013) 654-662.

[124] Q. Zhou, Y. Dong, H. Zhang, Y. Gao, The analytic hierarchy process with personalized individual semantics, Int. J. Comput. Intell. Syst. 11 (2018) 451.

[125] C.C. Li, Y. Dong, F. Herrera, E. Herrera-Viedma, L. Martínez, Personalized individual semantics in computing with words for supporting linguistic group decision making. An application on consensus reaching, Inf. Fusion. 33 (2017) 29-40.

[126] H. Huang, C. Li, S. Member, Extended personalized individual semantics with 2-tuple, IEICE Trans. Inf. Syst. 101 (2018) 387-395.

[127] C.C. Li, R.M. Rodríguez, F. Herrera, L. Martinez, Y. Dong, A consistency-driven approach to set personalized numerical scales for hesitant fuzzy linguistic preference relations, in: IEEE Int. Conf. Fuzzy Syst., 2017: pp. 1-5.

[128] F. Jin, Z. Ni, L. Pei, H. Chen, Z. Tao, X. Zhu, L. Ni, Approaches to group decision making with linguistic preference relations based on multiplicative consistency, Comput. Ind. Eng. 114 (2017) 69-79.

[129] F.J. Cabrerizo, R. Heradio, I.J. Pérez, E. Herrera-Viedma, A selection process based on additive consistency to deal with incomplete fuzzy linguistic information., J. Univers. Comput. Sci. 16 (2010) 
62-81.

[130] E. Herrera-Viedma, F.J. Cabrerizo, J. Kacprzyk, W. Pedrycz, A review of soft consensus models in a fuzzy environment, Inf. Fusion. 17 (2014) 4-13.

[131] I. Palomares, F.J. Estrella, L. Martínez, F. Herrera, Consensus under a fuzzy context: Taxonomy, analysis framework AFRYCA and experimental case of study, Inf. Fusion. 20 (2014) 252-271.

[132] I.J. Pérez, F.J. Cabrerizo, S. Alonso, Y.C. Dong, F. Chiclana, E. Herrera-Viedma, On dynamic consensus processes in group decision making problems, Inf. Sci. (Ny). 459 (2018) 20-35.

[133] Y. Dong, G. Zhang, W.-C. Hong, S. Yu, Linguistic computational model based on 2-tuples and intervals., IEEE Trans. Fuzzy Syst. 21 (2013) 1006-1018.

[134] C.C. Li, R.M. Rodríguez, L. Martínez, Y. Dong, F. Herrera, Personalized individual semantics based on consistency in hesitant linguistic group decision making with comparative linguistic expressions, Knowledge-Based Syst. 145 (2018) 156-165.

[135] Y. Dong, W.-C. Hong, Y. Xu, S. Yu, Selecting the individual numerical scale and prioritization method in the analytic hierarchy process: A 2-tuple fuzzy linguistic approach, IEEE Trans. Fuzzy Syst. 19 (2011) $13-25$.

[136] Y. Dong, Z.-P. Fan, S. Yu, Consensus building in a local context for the AHP-GDM with the individual numerical scale and prioritization method, IEEE Trans. Fuzzy Syst. 23 (2015) 354-368.

[137] W. Liu, Y. Dong, F. Chiclana, F.J. Cabrerizo, E. Herrera-Viedma, Group decision-making based on heterogeneous preference relations with self-confidence, Fuzzy Optim. Decis. Mak. 16 (2017) 429-447.

[138] Y. Dong, Q. Zha, H. Zhang, G. Kou, H. Fujita, F. Chiclana, E. Herrera-Viedma, Consensus reaching in social network group decision making: Research paradigms and challenges, Knowledge-Based Syst. (2018), DOI: 10.1016/j.knosys.2018.06.036.

[139] Y. Dong, M. Zhan, G. Kou, Z. Ding, H. Liang, A survey on the fusion process in opinion dynamics, Inf. Fusion. 43 (2018) 57-65.

[140] J.B. Lan, M.M. Hu, X.M. Ye, S.Q. Sun, Deriving interval weights from an interval multiplicative consistent fuzzy preference relation. Knowledge-Based Systems 26 (2012) 128-134.

[141] Y.J. Xu, K.W. Li, H.M. Wang, Consistency test and weight generation for additive interval fuzzy preference relations, Soft Computing 18 (2014) 1499-1513.

[142] W.W. Koczkodaj, R. Urban, Axiomatization of inconsistency indicators for pairwise comparisons, Int. J. Approx. Reason. 94 (2018) 18-29.

[143] M. Brunelli, M. Fedrizzi, Axiomatic properties of inconsistency indices for pairwise comparisons, J. Oper. Res. Soc. 66 (2015) 1-15.

[144] L. Csató, Characterization of an inconsistency ranking for pairwise comparison matrices, Ann. Oper. Res. 261 (2018) 155-165.

[145] F. Chiclana, E. Herrera-Viedma, S. Alonso, F. Herrera, Cardinal consistency of reciprocal preference relations: a characterization of multiplicative transitivity, IEEE Trans. Fuzzy Syst. 17 (2009) 14-23.

[146] Y.J. Xu, F. Herrera, Visualizing and rectifying different inconsistencies for fuzzy reciprocal preference relations, Fuzzy Sets and Systems, (2018), DOI: 10.1016/j.fss.2018.09.009. 